\title{
On the Existence of Bayesian Cournot Equilibrium*
}

\author{
Ezra Einy $^{1}$, Ori Haimanko², Diego Moreno ${ }^{3}$, and Benyamin Shitovitz ${ }^{4}$
}

October $2008^{* *}$

1 Graduate School of Economics, Hitotsubashi University

Naka 2-1, Kunitachi, Tokyo 186-8601, Japan (einy@econ.hit-u.ac.jp);

and Department of Economics, Ben Gurion University of the Negev

Beer Sheva 84105, Israel (einy@bgu.ac.il)

2 Department of Economics, Ben Gurion University of the Negev

Beer Sheva 84105, Israel (orih@bgu.ac.il)

3 Departamento de Economía, Universidad Carlos III de Madrid

28903 Getafe, Spain (dmoreno@eco.uc3m.es)

4 Department of Economics, University of Haifa

Haifa 31905, Israel (binya@econ.haifa.ac.il)

*This is a revised version of Working Paper 07-02 of Monaster Center for Economic Research.

${ }^{* *}$ Previous version: January 2008.

Acknowledgements: The authors are indebted to an anonymous referee for insightful suggestions that greatly improved the presentation of our results. These suggestions helped us both to better position our work in the general context, and to provide a sharper insight into the source of non-existence of a Bayesian Cournot equilibrium in the examples presented in Section 3.

The authors also gratefully acknowledge financial support of the Spanish Ministry of Education, grant SEJ2007-67436. 


\begin{abstract}
We show that when firms have incomplete information about the market demand and their costs, a (Bayesian) Cournot equilibrium in pure strategies may not exist, or be unique. In fact, we are able to construct surprisingly simple and robust examples of duopolies with these features. However, we also find some sufficient conditions for existence, and for uniqueness, of Cournot equilibrium in a certain class of industries. More general results arise when negative prices are possible.
\end{abstract}

Keywords: Oligopoly, Incomplete Information, Bayesian, Cournot, Equilibrium, Existence, Uniqueness

Journal of Economic Literature Classification Numbers: C72, D43, L13. 


\section{Introduction}

The Cournot model is widely used in studies of imperfectly competitive industries. Its standard version, which is concerned with the case of firms producing a homogeneous good with complete information about demand and production costs, has been extensively studied. However, in the past thirty years a fairly big amount of research has been dedicated to questions that arise when the information is incomplete, i.e., when there is uncertainty about the market demand and/or the firms' cost functions, and firms have asymmetric information about them. (See, e.g., Gal-Or (1985, 1986), Raith (1996), Sakai (1985, 1986), Shapiro (1986), Vives (1984, 1988, 1999), and Einy et al $(2002,2003)$.)

In oligopolies with incomplete information, some of the questions that have been addressed concern the value of information to a firm (that is, whether and by how much a firm can benefit from receiving additional information), as well as firms' incentives to share information. Treating these questions involves comparisons of the pure strategy Bayesian Cournot equilibrium outcomes in industries that differ with respect to the information endowments of the firms. The scope of these exercises is thus limited to classes of industries for which an equilibrium exists. ${ }^{1}$ Moreover, sharp and general conclusions are hard to obtain unless an equilibrium is also unique under various information endowments of the firms.

For a complete information oligopoly, the conditions for existence of a Cournot equilibrium that are found following the topological approach guarantee that the best response correspondences of the firms are closed graphed and convex-valued. For instance, if the inverse demand is a decreasing concave function, and firms' cost functions are convex, then a firm's payoff is a concave function of its own output, and under standard continuity assumptions the Nash Theorem yields equilibrium existence (see Szidarovszky and Yakowitz (1977)). In general, mere quasi-concavity of each firm's payoff in its own output and continuity of firms' payoff functions suffice to obtain existence.

An alternative approach to establishing existence of a Cournot equilibrium in the

\footnotetext{
${ }^{1}$ Throughout the paper we restrict attention to pure strategy equilibria. Existence of a mixed strategy Cournot equilibrium in an oligopoly does not present a problem under either complete or incomplete information (see remark 6 in Einy et al (2007)), and will not be taken as an issue here.
} 
complete information case is to study conditions under which firms' output decisions are strategic substitutes, i.e., the best response correspondence of a firm is decreasing in the output of the other firms. This feature together with the aggregation property of firms' payoffs (i.e., a firm's payoff depends only on its own output and on the aggregate output of all other firms) imply that the composite best response correspondence has a fixed point. This approach was pioneered by Novshek (1985), who establishes existence of equilibrium under a quite general condition on the inverse demand, and with minimal assumptions (monotonicity and continuity) on costs. Novshek's condition on the inverse demand requires that the marginal revenue of a firm be a decreasing function of the aggregate output of the other firms, and this indeed implies that firms' outputs are strategic substitutes.

Novshek's work spurred later developments (see Vives (1990), Kukushkin (1994), Amir (1996)), which adopted the lattice-theoretic framework and tools to study conditions for strategic substitutes and for existence of fixed points. (We may thus term this method of establishing equilibrium existence the lattice approach.) Vives (1990) noted that Novshek's condition is equivalent to the (cardinal) submodularity of the payoff function of each firm in its own output and on the aggregate output of other firms. Amir (1996) shows that log-concavity of the inverse demand ensures by itself that payoff functions are (ordinally) submodular ${ }^{2}$. Since both types of submodularity imply strategic substitutes, existence of equilibrium in a duopoly can be established using Tarski's fixed point theorem. ${ }^{3}$ Existence of equilibrium in oligopolies with more than two firms can be established using a more sophisticated fixed-point theorem due to Kukushkin (1994).

In oligopolies with incomplete information, the issues of existence of a (pure strategy) Bayesian Cournot equilibrium have been largely bypassed in the literature by making strong assumptions. For instance, Gal-Or (1985), Vives (1984, 1988), and Raith (1999) assume that the market demand is uncertain and linear, and allow the possibility that negative prices may arise for large outputs, in order not to break the linearity of the demand function. In other works (see, e.g., Sakai (1985)), incomplete information is assumed only on firms' linear costs, which again allows to avoid the

\footnotetext{
${ }^{2}$ More preceisely, each firm's payoff function satisfies the condition of reverse single crossing property of Amir (1996) with respect to its own output and the aggregate output of the other firms.

${ }^{3}$ See, theorems 1.1 and 1.2, and corollary 2.2, respectively, in Amir (1996).
} 
general problem of equilibrium existence. In a non-linear setting, Einy et al (2003) derive conditions under which the value of public information in an oligopoly is either positive or negative, but assume that the firms are symmetrically informed, which allows to reduce the equilibrium existence question to that in a complete information oligopoly. The assumption of symmetry of information, and a reduction to the complete information case that it allows, also stand behind the existence result of Lagerlöf (2006). In Einy et al (2002) a categorical approach is used: it is assumed that an equilibrium exists, and then its properties are investigated.

In this work we study conditions under which a Bayesian Cournot equilibrium exists, and is unique, in an oligopoly with incomplete information. As noted, quasiconcavity and ordinal submodularity are two very general properties of the payoff functions that are conducive to equilibrium existence in the complete information case. In the incomplete information scenario, however, if these properties are assumed to hold state-by-state, then they do not usually imply the corresponding properties of the expected payoff function. We thus confine ourselves to conditions on the primitives of the model that imply stronger properties of the payoff functions in each state (convexity or cardinal submodularity), at least on a certain range of output levels.

We will assume that prices are non-negative in every state of nature. (A model in which prices may be negative in some states of nature is less appealing; in section 4, however, we consider this case as well.) Requiring that prices be always nonnegative is far from being just a modelling nuance. Rather, it may change the strategic interaction in the oligopoly in a crucial way, and may lead to equilibrium non-existence even in well-behaved industries.

We present examples of duopolies with differential information without a Bayesian Cournot equilibrium, where, notably, the market demand and cost functions in every state of nature have properties that under complete information would have led to equilibrium existence. In our examples 1 and 2 the inverse demand is a decreasing linear or concave function in each state of nature, which is truncated where it intercepts the horizontal axis so as to preserve the non-negativity of prices; the costs are linear too. In addition, the information structure is very simple: one firm is better informed than the other. Yet, these industries possess no Bayesian Cournot equilibrium. 
The reason for equilibrium non-existence in our examples is the following. Without truncating the demand, we would have had concavity of the expected payoff function of each firm in its output, and existence of equilibrium could have been established by standard arguments. However, with a truncated demand, the payoff functions in each state of nature are only quasi-concave. This would have sufficed to obtain equilibrium existence in the complete information case, but with incomplete information quasiconcavity of the state-dependent payoff functions does not necessarily translate into the same property of the expected payoff function, as was mentioned already. Indeed, in our examples the expected payoff function of the least informed firm is not quasiconcave. In both examples the demands can be approximated by smooth curves that do not intersect the horizontal axis, with the expected payoff function remaining non-quasi-concave.

In the industries defined in examples 1 and 2 a Bayesian Cournot equilibrium exists if the demand is not truncated (and thus negative prices are possible) ${ }^{4}$. In terms of firms' incentives, the reason why these equilibria cease to be such when the demand is truncated is simple: whereas deviations from equilibrium to large outputs are deterred by the possibility of negative prices, when the demand is truncated such deviations may become profitable. Indeed, if a firm has high-demand and low-demand states in a certain information set, and the possibility of negative prices is ruled out, it is sometimes profitable to increase output in that information set. This deviation will increase revenue in high-demand states, but lead only to limited losses in low-demand states, where the revenue cannot fall below zero.

The simplicity and robustness of examples 1 and 2 indicate that Bayesian Cournot equilibrium existence with always non-negative prices is a much scarcer phenomenon than existence when negative prices are allowed to arise (and thus also scarcer than the existence in the complete information case $)^{5}$. When negative prices are possible

\footnotetext{
${ }^{4}$ Despite this possibility, prices in an equilibrium are positive in every state of nature.

${ }^{5}$ Note that in the complete information scenario, truncation of the inverse demand does not affect equilibrium existence, since the firms know where prices are positive and hence their best responses only lead to aggregate outputs corresponding to positive prices, or are zero (assuming positive marginal costs). Thus, only the positive, untruncated, part of the inverse demand function is relevant, and the firms' best responses are the same with or without truncation. All results on existence in the complete information case therefore apply equaly to truncated or non-truncated inverse demand.
} 
(which is admittedly somewhat less interesting), equilibrium exists quite often. In this case, linearity or concavity of the inverse demand, or more generally Novshek (1985) condition or concavity of the monopoly revenue, can be assumed to hold for all possible aggregate ouputs at every state of nature, which guarantees a good behavior of the expected payoff functions as well (e.g., concavity in each firm's strategy, or cardinal submodularity ${ }^{6}$ ). This can be used to establish equilibrium existence in the possibly negative prices scenario by arguments similar to those of the topological and lattice approaches in the complete information case, as we do in our theorems $1 \mathrm{~A}$ and $1 \mathrm{~B}$.

If one insists on always non-negative prices, then, typically, linearity or concavity of the inverse demand or Novshek's condition cannot hold on the entire $\mathbb{R}_{+}$. Imposing these conditions only until the state-dependent inverse demand reaches zero, and then truncating it, is but one way to guarantee always non-negative prices. Other adjustments to the above conditions that would leave the prices always non-negative are of course conceivable, but in all cases the expected payoff functions are likely to lose properties conducive to equilibrium existence, compared to the scenario of possibly negative prices (just as it occurs in oligopolies in examples 1 and 2). Nonetheless, we characterize a certain class of oligopolies with incomplete information in which a Bayesian Cournot equilibrium does exist - see theorems 2A, 2B and Corollary 1. The key feature of this class is the existence of certain thresholds of output which no firm will ever desire to exceed, and which guarantee positive prices in every state of nature if firms adhere to them. (Existence of such thresholds is guaranteed if firms' marginal costs increase sufficiently fast.)

On the front of uniqueness, it turns out that even a simple duopoly may have multiple Bayesian Cournot equilibria - see example 4. However, we show that in an oligopoly with two types of firms in which one type has superior information, whenever a Bayesian Cournot equilibrium exists, it must be unique - see theorems

\footnotetext{
${ }^{6}$ These cardinal properties, if satisfied in every state by the state-dependent payoff function, are preserved by the expectation operator, and thus the expected payoff functions inherit them. As we noted above, this is not the case with quasi-concavity. Similarly, we do not consider Amir (1996) condition of log-concavity of the inverse demand at each state of nature as a possible replacement/generalization of the Novshek (1986) condition, since ordinal submodularity of the payoff function in each state that it implies is not necessarily preserved by the expectation operator.
} 
$1 \mathrm{C}$ and $2 \mathrm{C}$.

The rest of the paper is organized as follows. Section 2 describes the set-up. Section 3 contains examples of oligopolies with incomplete information that possess no Bayesian Cournot equilibrium. Section 4 presents equilibrium existence and uniqueness results when possibly negative prices are allowed in the oligopolies, while Section 5 is dedicated to general results in oligopolies with always non-negative prices. Proofs of all results are given in the appendix.

\section{Cournot Competition with Incomplete Informa- tion}

Consider an industry where a set of firms, $N=\{1,2, \ldots, n\}$, compete in the production of a homogeneous good. There is uncertainty about the market demand and the production costs. This uncertainty is described by a finite set $\Omega$ of states of nature, together with a probability measure $\mu$ on $\Omega$, which represents the common prior belief of the firms about the distribution of the realized state. ${ }^{7}$ The information of the firms about the state of nature may be incomplete: the private information of firm $i \in N$ is given by a partition $\Pi^{i}$ of $\Omega$ into disjoint sets. For any $\omega \in \Omega, \Pi^{i}(\omega)$ denotes the information set of $i$ given $\omega$, that is, the element of $\Pi^{i}$ that contains $\omega$. W.l.o.g., we assume that $\mu$ has full support on $\Omega$, that is, $\mu\left(\Pi^{i}(\omega)\right)>0$ for every $i \in N$ and $\omega \in \Omega$.

If $q^{i}(\omega)$ denotes the quantity of the good produced by firm $i$ in state $\omega \in \Omega$, and $Q(\omega) \equiv \sum_{i=1}^{n} q^{i}(\omega)$ is the aggregate output in $\omega$, then the profit of firm $i$ in $\omega$ is given by

$$
u^{i}\left(\omega,\left(q^{1}(\omega), \ldots, q^{n}(\omega)\right)\right)=q^{i}(\omega) P(\omega, Q(\omega))-c^{i}\left(\omega, q^{i}(\omega)\right),
$$

where $P(\omega, \cdot)$ is the inverse demand function in $\omega$, and $c^{i}(\omega, \cdot)$ is the cost function of firm $i$ in $\omega$.

We assume throughout that:

\footnotetext{
${ }^{7}$ The assumption that $\Omega$ is finite is not necessary, and is made only to simplify the presentation - see Remark 2.
} 
(i) For every $\omega \in \Omega$ and $i \in N, c^{i}(\omega, \cdot)$ is continuous, and satisfies $c^{i}(\omega, 0)=0$ (i.e., there are no fixed costs).

(ii) For every $\omega \in \Omega, P(\omega, \cdot)$ is non-increasing, and for every $\omega \in \Omega$ there exists a level of aggregate output $\bar{Q}(\omega) \in[0, \infty]$ such that for every $Q<\bar{Q}(\omega)$

$$
P(\omega, Q)>0,
$$

and

$$
P(\omega, \bar{Q}(\omega))=0
$$

if $\bar{Q}(\omega)<\infty$. When $\bar{Q}(\omega)$ is finite, it is referred to as the horizontal demand intercept in $\omega .^{8}$

(iii) There exists a level of output $Z<\infty$ such that for every $i \in N, q \geq Z$ and $\omega \in \Omega$

$$
q P(\omega, q)-c^{i}(\omega, q) \leq 0
$$

i.e., in every state of nature each firm's monopoly profit is non-positive when its output exceeds $Z$. If $\bar{Q}(\omega)<\infty$ for every $\omega \in \Omega$, and cost functions are non-decreasing, one may take $Z \equiv \max _{\omega \in \Omega} \bar{Q}(\omega)$. Also, if the monopoly revenue function $q P(\omega, q)$ has a maximum and the cost functions are strictly increasing and convex, such a $Z$ exists.

A (pure) strategy for firm $i$ is a function $q^{i}: \Omega \rightarrow \mathbb{R}_{+}$that specifies its output in every state of nature, subject to measurability with respect to $i$ 's private information (i.e., $q^{i}$ is constant on every information set of firm $i$ ). The set of strategies of firm $i$ will be denoted by $B\left(\Omega, \Pi^{i}\right)$. Given a strategy profile $q=\left(q^{1}, \ldots, q^{n}\right) \in \prod_{j=1}^{n} B\left(\Omega, \Pi^{j}\right)$ the expected profit of firm $i$ is

$$
U^{i}(q)=E\left[u^{i}\left(\cdot,\left(q^{1}(\cdot), \ldots, q^{1}(\cdot)\right)\right)\right]
$$

A strategy profile $q_{*} \in \prod_{j=1}^{n} B\left(\Omega, \Pi^{j}\right)$ a (pure strategy Bayesian) Cournot equilibrium, if no firm finds it profitable to unilaterally deviate to another strategy, i.e., if for every $i \in N$ and $q^{i} \in B\left(\Omega, \Pi^{i}\right)$

$$
U^{i}\left(q_{*}\right) \geq U^{i}\left(q_{*} \mid q^{i}\right)
$$

\footnotetext{
${ }^{8} \mathrm{~A}$ demand intercept arises in standard complete information models with a linear or concave inverse demand function. Existence of a demand intercept is consistent with (and usually implied by) Novshek's condition - see Remark 5.1 in Novshek (1985).
} 
where $\left(q_{*} \mid q^{i}\right)$ stands for the profile of strategies which is identical to $q_{*}$ in all but the $i$ th strategy, which is replaced by $q^{i}$. This is equivalent to requiring

$$
E\left(u^{i}\left(\cdot, q_{*}(\cdot)\right) \mid \Pi^{i}(\omega)\right) \geq E\left(u^{i}\left(\cdot,\left(q_{*} \mid q^{i}\right)(\cdot)\right) \mid \Pi^{i}(\omega)\right)
$$

for every $\omega \in \Omega$. Here $E(g(\cdot) \mid A)$ stands for the expectation of a random variable $g$ conditional on event $A$.

\section{A Cournot Equilibrium May Not Exist: Exam- ples}

In this section we present two simple examples of duopolies with incomplete information for which a Cournot equilibrium does not exist. Example 1 concerns a duopoly where, in each state of nature, the firms' cost functions are linear, and the inverse market demand is a decreasing linear function which is truncated where it reaches zero, to ensure always non-negative prices. Surprisingly, when firms' have incomplete information, existence of a Bayesian Cournot equilibrium cannot be guaranteed even in this simple setting. (Note that in such a setting a Cournot equilibrium exists when firms have complete information.)

Equilibrium non-existence in example 1 is driven by the asymmetry in firms' information about the demand intercept $\bar{Q} \cdot{ }^{9}$ The linear inverse demand of example 1 is modified in example 2 (where it is piecewise linear and concave) to show that a Cournot equilibrium may fail to exist even if the intercept $\bar{Q}$ is known to both firms (in fact, in this example $\bar{Q}$ is the same in all states of nature).

Example 1. Consider the following duopoly with incomplete information. The set of states of nature $\Omega$ consists of just two states, $\omega_{1}$ and $\omega_{2}$. The probability of $\omega_{1}$ is $\frac{1}{4}$, and the probability of $\omega_{2}$ is $\frac{3}{4}$. Firm 1 is informed about the realized state of nature, while firm 2 has no information about it; i.e., $\Pi^{1}=\left\{\left\{\omega_{1}\right\},\left\{\omega_{2}\right\}\right\}$ and $\Pi^{2}=\{\Omega\}$. The inverse demand function is

$$
P\left(\omega_{i}, Q\right)=\max \left\{1-b\left(\omega_{i}\right) Q, 0\right\}
$$

\footnotetext{
${ }^{9}$ In fact, example 3 in section 5 shows that a linear duopoly where the demand intercept is known to both firms does possess a Cournot equilibrium.
} 
where $b\left(\omega_{1}\right)=\frac{1}{4}$ and $b\left(\omega_{2}\right)=1$. Thus, both $P\left(\omega_{1}, \cdot\right)$ and $P\left(\omega_{2}, \cdot\right)$ are linear till they reach zero, at which point they are truncated and set to be equal to zero. This ensures that the prices are always non-negative: in the state $\omega_{i}$ the inverse demand function $P$ is positive on $\left[0, \bar{Q}\left(\omega_{i}\right)\right)$, and is zero for $Q \geq \bar{Q}\left(\omega_{i}\right)$, where $\bar{Q}\left(\omega_{1}\right)=4$ and $\bar{Q}\left(\omega_{2}\right)=1$. The marginal costs of firm 1 are $c^{1}\left(\omega_{1}\right)=2$ and $c^{1}\left(\omega_{2}\right)=\frac{1}{100}$. Firm 2 has a constant marginal cost $c^{2}=\frac{1}{100}$ in both states of nature.

Since the marginal revenue of firm 1 in $\omega_{1}$ is always below its marginal cost, maximizing profits entails that firm 1 produces zero in this state. Thus, in looking for an equilibrium we restrict attention to those strategies of firm $1, q^{1} \in B\left(\Omega, \Pi^{1}\right)$, that prescribe producing zero in $\omega_{1}$; i.e., $q^{1}$ can be identified with a scalar $x \equiv q^{1}\left(\omega_{2}\right) \in \mathbb{R}_{+}$. Also, since firm 2 does not know the realized state, a strategy of firm $2, q^{2} \in B\left(\Omega, \Pi^{2}\right)$, must specify the same output in both states of nature; i.e., $q^{2}$ can be identified with a scalar $y \equiv q^{2}\left(\omega_{1}\right)=q^{2}\left(\omega_{2}\right) \in \mathbb{R}_{+}$. Accordingly, the strategies of firms 1 and 2 will be regarded as scalars $x, y \in \mathbb{R}_{+}$. By identifying firms' strategies with scalars $x, y$ we have in effect converted the incomplete information duopoly into a complete information game where the payoffs are the firms' expected profits.

In order to understand the source of the existence problem in this example, we begin by showing that if the demand functions are not truncated, and hence prices may be negative, then the industry has a Cournot equilibrium. We proceed then to modify the analysis to account for the impact of truncating the demand, and we show then that no Cournot equilibrium exists in the industry. Therefore, assume for the moment that the inverse demand is

$$
P_{-}\left(\omega_{i}, Q\right)=1-b\left(\omega_{i}\right) Q
$$

and for $(x, y) \in \mathbb{R}_{+}^{2}$ denote by $U_{-}^{i}(x, y)$ the corresponding payoff of firm $i \in\{1,2\}$. Since firm 1 only produces in $\omega_{2}$, we have

$$
U_{-}^{1}(x, y)=\frac{3}{4}\left(P_{-}\left(\omega_{2}, x+y\right) x-\frac{x}{100}\right) .
$$

The payoff of firm 2 is

$$
U_{-}^{2}(x, y)=\bar{P}_{-}^{x}(y) y-\frac{y}{100}
$$

where

$$
\bar{P}_{-}^{x}(y)=\frac{1}{4} P_{-}\left(\omega_{1}, y\right)+\frac{3}{4} P_{-}\left(\omega_{2}, x+y\right)
$$


is firm 2's "expected residual inverse demand."

Firms' reaction functions are

$$
R_{-}^{1}(y)=\max \left\{\frac{1}{2}\left(\frac{99}{100}-y\right), 0\right\},
$$

and

$$
R_{-}^{2}(x)=\max \left\{\frac{198}{325}-\frac{6}{13} x, 0\right\},
$$

and therefore $\left(x^{*}, y^{*}\right)=\left(\frac{99}{400}, \frac{99}{200}\right)$ is the unique Cournot equilibrium. Figure 1a shows the state-dependent residual inverse demand functions, and the expected residual inverse demand function, faced by firm 2. Figure $1 \mathrm{~b}$ shows the payoff of firm 2 given that firm 1 produces the equilibrium output $x^{*}=\frac{99}{400}$. Figure $1 \mathrm{c}$ shows firms' reaction functions.

Figure 1 goes here.

We modify now the analysis to account for the demand truncation, i.e., we revert from (5) to (4). Figure 2a below shows a graph of the residual inverse demand functions faced by firm 2 . The expected residual demand faced by firm 2 is now

$$
\bar{P}^{x}(y)=\frac{1}{4} P\left(\omega_{1}, y\right)+\frac{3}{4} P\left(\omega_{2}, x+y\right) ;
$$

i.e.,

$$
\bar{P}^{x}(y)= \begin{cases}\bar{P}_{-}^{x}(y), & \text { if } x+y<1 \\ \hat{P}(y), & \text { if } 1 \leq x+y \text { and } y \leq 4 \\ 0, & \text { if } y>4\end{cases}
$$

where

$$
\hat{P}(y)=\frac{1}{4}\left(1-\frac{y}{4}\right) .
$$

Note that $\bar{P}^{x}(y)$ is not a concave function. Firms' payoffs are given for $(x, y) \in \mathbb{R}_{+}^{2}$ by

$$
U^{1}(x, y)=\frac{3}{4}\left(P\left(\omega_{2}, x+y\right) x-\frac{x}{100}\right) .
$$

and

$$
U^{2}(x, y)=\bar{P}^{x}(y) y-\frac{y}{100} .
$$

Figure $2 \mathrm{~b}$ below shows the graph of $U^{2}(x, \cdot)$ for $x=99 / 100$. (The graph of $U^{2}(x, \cdot)$ has a similar form for every $x$.) Note that $U^{2}(x, \cdot)$ is non-quasi-concave, despite the 
fact that the state-dependent payoff functions are quasi-concave; and that it has two local maxima. The local maximum of $U^{2}(x, \cdot)$ on the left is given by

$$
\max _{y}\left\{P_{-}^{x}(y) y-\frac{y}{100}\right\}=\max _{y}\left\{U_{-}^{2}(x, y)\right\}=U_{-}^{2}\left(x, R_{-}^{2}(x)\right) .
$$

The local maximum of $U^{2}(x, \cdot)$ on the right is firm 2's maximum payoff when the price in state $\omega_{2}$ is zero, given by

$$
\max _{y}\left\{\hat{P}(y) y-\frac{y}{100}\right\}=\frac{144}{625} .
$$

The smallest solution to the equation

$$
U_{-}^{2}\left(x, R_{-}^{2}(x)\right)=\frac{144}{625}
$$

is $\bar{x}=\frac{33}{25}-\frac{8}{25} \sqrt{13} \approx 0.16622$. It is easy to see that for $x<\bar{x}$ the local maximum of $U^{2}(x, \cdot)$ on the left (i.e., $\left.y=R_{-}^{2}(x)\right)$ is the global maximum of $U^{2}$. For $x>\bar{x}$ the local maximum of $U^{2}(x, \cdot)$ on the right (i.e., $y=\frac{48}{25}$ ) is the global maximum of $U^{2}(x, \cdot)$ - that is, in this case firm 2 is better off choosing the output that maximizes its profit in $\omega_{1}$, letting the price be zero in $\omega_{2}$.

Firm 1's reaction functions is $R^{1}(y)=R_{-}^{1}(y)$, and firm 2's reaction correspondence is given by

$$
R^{2}(x)=\left\{\begin{array}{cc}
R_{-}^{2}(x), & \text { if } x \leq \bar{x} \\
\left\{R_{-}^{2}(\bar{x}), \frac{48}{25}\right\}, & \text { if } x=\bar{x} \\
\frac{48}{25} & \text { if } x \geq \bar{x} .
\end{array}\right.
$$

A graph of firms' reaction functions is given in Figure 2c below. The jump of $R^{2}$ at $\bar{x}$ is caused by the change of the global maximizer of $U^{2}(x, \cdot)$ from $y=R_{-}^{2}(x)$ to $y=\frac{48}{25}$. Thus, the best response correspondence $R^{2}$ is not convex-valued. As the graphs of $R^{1}$ and $R^{2}$ show, these functions do not cross, and therefore a Cournot equilibrium does not exist.

Figure 2 goes here.

In example 1, firm 2's lack of information about the demand intercept $\bar{Q}$ leads to a non-concave expected residual inverse demand function, as shown in figure 2a. As a result, the expected revenue of firm 2 is not quasi-concave in its own output, and its reaction correspondence is not convex-valued. This causes equilibrium non-existence. 
However, as we shall see in section 5, example 3, a linear duopoly where $\bar{Q}$ is known to both firms does possess a Cournot equilibrium.

Next we present an example of a duopoly in which the demand intercept $\bar{Q}$ is the same in all states of nature (and hence known to both firms), but nonetheless a Cournot equilibrium does not exist. This duopoly is a variation of that described in example 1: here the inverse demand function in $\omega_{1}, P\left(\omega_{1}, \cdot\right)$, is piecewise linear and concave on $[0, \bar{Q}]$. Unlike in example 1 , here the expected demand faced by firm 2 is concave on $[0, \bar{Q}]$ when the firm is a monopoly, i.e., when the output of firm 1 is zero. However, this does not extend to the expected residual demand in general. Despite the good properties of the inverse demand function, the expected residual inverse demand of firm 2 is not concave on $[0, \bar{Q}]$ for a considerable range of positive outputs of firm 1 - see figure 3a. As a result, the expected payoff function of firm 2 is not quasi-concave, see figure $3 \mathrm{~b}$, in its own output, which ultimately leads to non-existence of a Cournot equilibrium, see figure 3c.

Example 2. Consider an industry identical to that of example 1 except for the demand in $\omega_{1}$, which is given here by

$$
P\left(\omega_{1}, Q\right)=\left\{\begin{array}{cc}
1, & \text { if } Q \leq \frac{99}{100} \\
100(1-Q), & \text { if } \frac{99}{100}<Q \leq 1 \\
0, & \text { if } Q>1
\end{array}\right.
$$

The demand intercept is now constant, $\bar{Q}\left(\omega_{1}\right)=\bar{Q}\left(\omega_{2}\right)=1$, and thus known to both firms.

The expected residual inverse demand faced by firm $2, \bar{P}^{x}(y)$, is given by

$$
\bar{P}^{x}(y)= \begin{cases}\frac{1}{4}+\frac{3}{4}(1-x-y), & \text { if } x+y \leq 1 \text { and } y \leq \frac{99}{100} \\ \frac{1}{4} 100(1-y)+\frac{3}{4}(1-x-y), & \text { if } x+y \leq 1 \text { and } 1 \geq y>\frac{99}{100} \\ \frac{1}{4}, & \text { if } x+y>1 \text { and } y \leq \frac{99}{100} \\ \frac{1}{4} 100(1-y), & \text { if } x+y>1 \text { and } 1 \geq y>\frac{99}{100} \\ 0, & \text { otherwise }\end{cases}
$$

Note that for $\frac{1}{100}<x<1$ the function $\bar{P}^{x}(y)$ is not concave on $[0,1]$, even though the demand intercept is the same in both states. Figure 3a shows the graphs of the state-dependent residual inverse demands in that case, and the expected residual inverse demand. 
Let $(x, y) \in \mathbb{R}_{+}^{2}$. As in example 1 , since firm 1 produces zero in $\omega_{1}$ its payoff is

$$
U^{1}(x, y)=\frac{3}{4}\left(P\left(\omega_{2}, x+y\right) x-\frac{x}{100}\right) .
$$

Firm 2's payoff is

$$
U^{2}(x, y)=\bar{P}^{x}(y) y-\frac{y}{100} .
$$

Figure 3b show graphs of firm 2's expected payoff $U^{2}(x, \cdot)$ for $x=22 / 100$. Note that $U^{2}\left(\frac{22}{100}, \cdot\right)$ is not quasi-concave. (This stands in contrast to the case of firm 2 being a monopoly: $U^{2}(0, \cdot)$ is a quasi-concave function, which is, moreover, concave on $[0,1]$.)

The reaction function of firm 1 is also as in example 1, whereas firm 2's reaction function is now

$$
R^{2}(x)=\left\{\begin{array}{cc}
\frac{33}{50}-\frac{1}{2} x, & \text { if } x \leq \bar{x} \\
\left\{\frac{33}{50}-\frac{1}{2} \bar{x}, \frac{99}{100}\right\}, & \text { if } x=\bar{x} \\
\frac{99}{100}, & \text { if } x \geq \bar{x}
\end{array}\right.
$$

for $x \in \mathbb{R}_{+}$. Here $\bar{x}=\frac{33}{25}-\frac{6}{25} \sqrt{22} \approx 0.1943$ is the smallest solution of the equation

$$
\max _{y}\left\{\left(\frac{1}{4}+\frac{3}{4}(1-x-y)\right) y-\frac{y}{100}\right\}=\max _{y \in\left[0, \frac{99}{100}\right]}\left\{\frac{1}{4} y-\frac{y}{100}\right\} .
$$

Figure 3c shows the graphs of firms' reaction functions. Since firms' reaction functions do not cross, a Cournot equilibrium does not exists in this industry.

Figure 3 goes here

In both examples 1 and 2, the demand functions can be made smooth, and such that they do not intersect the horizontal axis in either state, while preserving the form of firm 2 expected profit function. These examples are suggestive of the difficulty in finding natural conditions on the primitives of the model, analogous to those found for the complete information case, that guarantee existence of a Cournot equilibrium when information is incomplete.

\section{Cournot Equilibrium when Negative Prices are Possible}

In this section we show existence of Cournot equilibrium under two assumptions which are standard in the complete information framework (see Theorems $1 \mathrm{~A}$ and 
1B below). These assumptions do not rule out the possibility of negative prices in some states of nature for sufficiently large aggregate outputs, and this possibility can play an important role in guaranteeing equilibrium existence. Indeed, as was pointed out in example 1, a Cournot equilibrium exists in the linear oligopoly described there if the inverse demand is not truncated at zero and thus allowed to receive negative values. Similarly, not truncating the concave inverse demand function in example 2 would also lead to equilibrium existence.

Although the possibility of negative prices might not be very meaningful in most contexts, this section is still valuable because its results can also be applied to certain classes of strictly positive inverse demand functions - see remark 1. Furthermore, the results presented here (and their proofs) will be instrumental in the next Section 5, where conditions of this section are combined with an explicit requirement of always non-negative prices.

The following condition on the inverse demand is akin to the collation of (2) and (3) in theorem 3 in Novshek (1985):

(A) For every $\omega \in \Omega, P(\omega, \cdot)$ is twice continuously differentiable and satisfies

$$
Q P^{\prime \prime}(\omega, Q)+P^{\prime}(\omega, Q) \leq 0
$$

for every $Q \in \mathbb{R}_{+}$. (At $Q=0$ we have in mind the right-side derivatives of $P$ and $P^{\prime}$.)

Inequality (9) in condition $A$ is equivalent to the requirement that the marginal revenue of a firm be decreasing in the aggregate output of the other firms. It is satisfied, e.g., by all decreasing, concave, and twice continuously differentiable inverse demand functions.

Theorem 1A. A duopoly that satisfies (i)-(iii) and $A$ has a Cournot equilibrium.

When the firms' cost functions have additional standard properties, we can consider condition $B$ below, which is weaker than $A$ (since the inverse demand is a non-increasing function).

$(B)$ For every $\omega \in \Omega, P(\omega, \cdot)$ is twice continuously differentiable and the monopoly revenue function $Q P(\omega, Q)$ is concave, i.e.,

$$
Q P^{\prime \prime}(\omega, Q)+2 P^{\prime}(\omega, Q) \leq 0
$$


for every $Q \in \mathbb{R}_{+}$.

Theorem 1B. An oligopoly that satisfies (i)-(iii) and $B$, and in which $c^{i}(\omega, \cdot)$ is convex for every $i \in N$ and $\omega \in \Omega$, has a Cournot equilibrium.

Theorems 1A and 1B are natural counterparts of results on existence of Cournot equilibrium that have been obtained in the literature in the complete information case (see, e.g., Novshek (1985) and Szidarovszky and Yakowitz (1977)). It is the nice behavior of the expected payoff functions that stands behind equilibrium existence in our theorems. Indeed, the proofs reveal that under condition $A$ the profit function of each firm is cardinally submodular in its output and the aggregate output of the other firms, and if the firms' cost functions are convex (as in theorem 1B), then the expected profit function of each firm is concave in its strategy - see section 6.1 in the appendix. Both concavity and cardinal submodularity imply existence of a Cournot equilibrium via known methods (that we dubbed the "lattice" and the "topological" approaches in the Introduction).

Theorem 1A considers only duopolies. This is because it is not known whether cardinal submodularity of the expected payoff functions implies equilibrium existence when there are more than two firms (except when the strategy sets are onedimensional, see Kukushkin (1994)). However, when convexity of cost functions is assumed, as in theorem 1B, existence of a Cournot equilibrium is guaranteed for any number of firms.

As was already mentioned, any non-increasing, twice continuously differentiable and concave $P(\omega, \cdot)$ satisfies condition $A$, and thus satisfies condition $B$ too. It might be tempting to contemplate an alternative to $A$ or $B$ that would require that each $P(\omega, \cdot)$ be log-concave. Such condition is used by Amir (1996) to prove existence of Cournot equilibrium in a duopoly when information is complete. However, this does not appear to be a viable alternative, since the log-concavity of $P(\omega, \cdot)$ only implies the reverse single crossing property of the state-dependent revenue function, and this property would not typically be passed to the expected revenue function.

With a decreasing and concave $P(\omega, \cdot)$, prices are negative for aggregate outputs exceeding $\bar{Q}(\omega)$. In fact, condition $A$ usually implies (see Remark 5.1 in Novshek (1985)) the existence of a finite demand intercept $\bar{Q}(\omega)$ such that prices are negative for aggregate outputs exceeding it. Theorems $1 \mathrm{~A}$ and $1 \mathrm{~B}$ may thus appear to be of 
limited interest. However, Remark 1 identifies an interesting class of inverse demand functions that satisfy $B$ (albeit not the stronger $A$ ) and are strictly positive. In section 5 we consider explicitly the implications of imposing the condition that the inverse demand be a non-negative function.

Remark 1. The class of inverse demand functions of the form

$$
P(\omega, Q)=\left(\frac{b(\omega)}{Q+c(\omega)}\right)^{a(\omega)}
$$

where $0<a(\omega)<1$ and $b(\omega), c(\omega)>0$ for all $\omega \in \Omega$, satisfy condition $B$. These functions, which are often used in applications, are strictly positive.

Condition $A$, combined with assumptions on the inverse demand and cost functions that are stronger than our maintained assumptions (i) and (ii), allows us to establish uniqueness of Cournot equilibrium for certain information structures. Specifically, we assume that there are two types of firms, one of which possesses superior information (i.e., has a finer information partition). Condition $U$ formalizes this assumption:

$(U)$ The set $N$ of firms can be partitioned into two disjoint sets, $K$ and $M$, such that $1 \in K, 2 \in M$, and such that $\Pi^{i}=\Pi^{1}, c^{i}=c^{1}$ for every $i \in K, \Pi^{j}=\Pi^{2}, c^{j}=c^{2}$ for every $j \in M$, and $\Pi^{1}$ is finer than (or equal to) $\Pi^{2}$.

Theorem 1C. Consider an oligopoly satisfying conditions (i), (iii), $A$ and $U$, and such that for every $\omega \in \Omega, P(\omega, \cdot)$ is strictly decreasing, and each $c^{i}(\omega, \cdot)$ is strictly increasing, twice continuously differentiable and convex for $i \in N$. Then it has a unique Cournot equilibrium.

Even though it is possible to dispose off assumption $U$ following the arguments of the proof of theorem 2 in Hon-Snir et al (2007), we chose to present here a weaker version of the uniqueness result, since its proofs extends easily to the case of non-negative inverse demand functions considered in section 5. In this latter case existence of superior information is indispensable for equilibrium uniqueness, as will be demonstrated in example 4. 


\section{Cournot Equilibrium with Always Non-Negative Prices}

In the previous section we established existence of a Cournot equilibrium under conditions $A$ or $B$. Although we presented (in remark 1) a class of oligopolies with positive demand functions for which $B$ holds, it is common for a smooth inverse demand function satisfying $B$ (and even more so for a demand satisfying $A$ ) to become negative for sufficiently large levels of aggregate output. (This is also the case in the complete information setting - see remark 5.1 in Novshek (1985).) If the inverse demand is then truncated to rule out non-negative prices, existence of a Cournot equilibrium cannot be guaranteed even in duopolies with linear or concave inverse demand functions, as seen in examples 1 and 2.

In this section we discuss the case of non-negative inverse demand functions, and present additional existence and uniqueness results. The simplicity of the demand functions in examples 1 and 2 indicates that it is difficult to find natural conditions on the primitives of the oligopoly that would guarantee equilibrium existence. Our existence theorems $2 \mathrm{~A}$ and $2 \mathrm{~B}$ and corollary 1 rely on conditions stated directly as properties of firms' profit functions.

Consider a non-negative inverse demand function $P$, i.e., a function that satisfies $P(\omega, Q) \geq 0$ for all $\omega \in \Omega$ and $Q \in \mathbb{R}_{+}$. Since for every $\omega \in \Omega, P(\omega, \cdot)$ is nonincreasing by assumption (ii), for $Q \geq \bar{Q}(\omega)$ we have

$$
P(\omega, Q)=0
$$

(Recall that $\bar{Q}(\omega)$ is the horizontal demand intercept.) Of course, we may have $\bar{Q}(\omega)=\infty$, as in the class of inverse demand functions described in remark 1. But, if $\bar{Q}(\omega)$ is finite, then (11) implies that the inverse demand is fixed at zero beyond the demand intercept $\bar{Q}(\omega)$.

The analogs of Novshek's condition $A$, or the revenue concavity condition $B$, will now be used in conjunction with the requirement that the inverse demand be a non-negative function. These conditions must now be restated in the form that makes them consistent with (11). (In what follows, the conditions and assumptions on derivatives of $P(\omega, \cdot)$ refer to one-sided derivatives at the endpoints of the interval 
$\left.[0, \bar{Q}(\omega)] \cdot{ }^{10}\right)$

$\left(A^{\prime}\right)$ For every $\omega \in \Omega, P(\omega, \cdot)$ is a non-negative function that is twice continuously differentiable on $[0, \bar{Q}(\omega)]$ and satisfies $Q P^{\prime \prime}(\omega, Q)+P^{\prime}(\omega, Q) \leq 0$ for every $Q \in$ $[0, \bar{Q}(\omega)]$.

$\left(B^{\prime}\right)$ For every $\omega \in \Omega, P(\omega, \cdot)$ is a non-negative function that is twice continuously differentiable on $[0, \bar{Q}(\omega)]$ and satisfies $Q P^{\prime \prime}(\omega, Q)+2 P^{\prime}(\omega, Q) \leq 0$ for every $Q \in$ $[0, \bar{Q}(\omega)]$.

The following condition is used in theorems $2 \mathrm{~A}$ and $2 \mathrm{~B}$ below.

(C) There exists a profile of state-dependent thresholds of output $\bar{q} \in \prod_{i=1}^{n} B\left(\Omega, \Pi^{i}\right)$ such that for every $\omega \in \Omega$

$$
\sum_{i=1}^{n} \bar{q}^{i}(\omega) \leq \bar{Q}(\omega)
$$

and for every strategy profile $q \in \prod_{i=1}^{n} B\left(\Omega, \Pi^{i}\right)$ and every $i \in N$ there exists a strategy $r^{i} \leq \bar{q}^{i}$ such that ${ }^{11}$

$$
U^{i}(q) \leq U^{i}\left(q \mid r^{i}\right)
$$

Intuitively, condition $C$ implies that each firm $i$ does not want to produce too much, since by reducing its output below the level $\bar{q}^{i}$ its expected profit does not decrease.

The following two results are the counterparts of theorems $1 \mathrm{~A}$ and $1 \mathrm{~B}$ in section 4, stated now for oligopolies with always non-negative prices.

Theorem 2A. A duopoly satisfying conditions (i)-(iii), $A^{\prime}$ and $C$ has a Cournot equilibrium.

Theorem 2B. An oligopoly satisfying conditions (i)-(iii), $B^{\prime}$ and $C$, and such that $c^{i}(\omega, \cdot)$ is convex for every $i \in N$ and $\omega \in \Omega$, has a Cournot equilibrium.

\footnotetext{
${ }^{10}$ The function $P(\omega, \cdot)$ need not (and typically will not) be differentiable at $\bar{Q}(\omega)$. If $\bar{Q}(\omega)=\infty$ then the inequality is assumed on the entire $\mathbb{R}_{+}$, i.e., conditions $A^{\prime}$ or $B^{\prime}$ become $A$ or $B$, respectively.

${ }^{11}$ Here and henceforth, we use the notation $h \leq g$ (for $h, g: \Omega \rightarrow \mathbb{R}_{+}$) if and only if $h(\omega) \leq g(\omega)$ for every $\omega \in \Omega$.
} 
When firms produce below the thresholds $\left(\bar{q}^{i}\right)_{i=1}^{n}$ in their best responses, as implied by condition $C$, prices are positive due to (12) and deviations are evaluated in the domain where the inverse demand function is twice continuously differentiable and obeys inequality (9) of condition $A$ or (10) of condition $B$. This allows us to establish existence of a Cournot equilibrium using arguments analogous to those of Section 4 , where (9) or (10) hold on the entire $\mathbb{R}_{+}$.

Theorems $2 \mathrm{~A}$ and $2 \mathrm{~B}$ lead to the following corollary, that establishes existence of a Cournot equilibrium if there are thresholds $\left(\bar{q}^{i}\right)_{i=1}^{n}$ satisfying (12) such that the expected monopoly profit of any firm $i$ under any strategy exceeding $\bar{q}^{i}$ is nonpositive, given $i$ 's information. Denote by $0^{-i}$ the profile of strategies of all firms but $i$ according to which every firm produces zero in every state of nature:

Corollary 1. There exists a Cournot equilibrium in an oligopoly satisfying the assumptions of either theorem $1 \mathrm{~A}$ or theorem $1 \mathrm{~B}$, if condition $C$ is replaced by the following: there exists $\bar{q} \in \prod_{i=1}^{n} B\left(\Omega, \Pi^{i}\right)$ such that $\sum_{i=1}^{n} \bar{q}^{i}(\omega) \leq \bar{Q}(\omega)$, and

$$
E\left(u^{i}\left(\cdot,\left(q^{i}, 0^{-i}\right)\right) \mid \Pi^{i}(\omega)\right) \leq 0
$$

for every $i \in N$, every $\omega \in \Omega$ and every strategy $q^{i}$ that exceeds $\bar{q}^{i}$ on $\Pi^{i}(\omega)$.

In example 3 below we apply theorem $2 \mathrm{~A}$ to a duopoly with linear demand, and show that a Cournot equilibrium exists provided the demand intercept $\bar{Q}$ is known to both firms.

Example 3 (A duopoly with a linear demand and complete information on the demand intercept). Suppose that $n=2$. Let $\alpha, \beta: \Omega \rightarrow \mathbb{R}_{++}$be strictly positive functions. Assume that $\beta \in B\left(\Omega, \Pi^{1}\right) \cap B\left(\Omega, \Pi^{2}\right)$, where $\Pi^{1}$ and $\Pi^{2}$ are information endowments of the duopolists. Suppose that for any $\omega \in \Omega$

$$
P(\omega, Q)=\max \{\alpha(\omega)(\beta(\omega)-Q), 0\},
$$

and that the cost functions satisfy (i) and are non-decreasing. Here $\bar{Q}=\beta$. Since $\beta$ is both $\Pi^{1}$ - and $\Pi^{2}$-measurable, both firms know the demand intercept in every state of nature. This is a crucial difference with example 1 , where $\bar{Q}$ was not measurable with respect to the information partition of firm 2, and a Cournot equilibrium does 
not exist. Here, the measurability $\bar{Q}=\beta$ with respect to both partitions leads to a different conclusion.

Let $\bar{q}^{1}=\bar{q}^{2} \equiv \frac{1}{2} \beta \in B\left(\Omega, \Pi^{1}\right) \cap B\left(\Omega, \Pi^{2}\right)$. Clearly $\left(\bar{q}^{1}, \bar{q}^{2}\right)$ satisfies (12) of condition $C$. But $\frac{1}{2} \beta$ is the revenue maximizing monopoly output level, since the firms know $\beta$ and the demand is linear on $[0, \beta]$, and thus no firm will exceed $\frac{1}{2} \beta$ in any best response. Therefore condition $C$ holds, and the duopoly has a Cournot equilibrium by theorem $2 \mathrm{~A}$.

The following theorem is a counterpart to theorem $1 \mathrm{C}$ when the inverse demand is a non-negative function. It establishes conditions that guarantee that, when a Cournot equilibrium exists, it is also unique.

Theorem 2C. Consider an oligopoly satisfying conditions (i), (iii), $A^{\prime}$ and $U$. Also assume that for every $\omega \in \Omega, P(\omega, \cdot)$ is strictly decreasing on $[0, \bar{Q}(\omega)]$, where $\bar{Q}$ is a strictly positive and $\Pi^{2}$-measurable function, ${ }^{12}$ and each $c^{i}(\omega, \cdot)$ is strictly increasing, twice continuously differentiable and convex for $i \in N$. If a Cournot equilibrium exists (e.g., under conditions of theorems $2 \mathrm{~A}$ or $2 \mathrm{~B}$ ), then it is unique.

In example 3 we established existence of a Cournot equilibrium for a duopoly with linear demand, provided the demand intercept $\bar{Q}$ is known to both firms. If in addition we assume that $\Pi^{1}$ is finer than $\Pi^{2}$, and that the state-dependent costs are strictly increasing, twice continuously differentiable and convex, then by theorem $2 \mathrm{C}$ this duopoly's Cournot equilibrium is unique. The next example shows that in theorem $2 \mathrm{C}$ we cannot dispense with condition $U$.

Example 4 (Non-Uniqueness of Cournot Equilibrium when no Firm Has Superior Information). Consider a duopoly in which $\Omega$ consists of three states, $\omega_{1}, \omega_{2}$, and $\omega_{3}$; each one is chosen by nature with equal probability. Firms' information partitions are $\Pi^{1}=\left\{\left\{\omega_{1}, \omega_{2}\right\},\left\{\omega_{3}\right\}\right\}$, and $\Pi^{2}=\left\{\left\{\omega_{1}, \omega_{3}\right\},\left\{\omega_{2}\right\}\right\}$; i.e., firm 1 cannot distinguish between $\omega_{1}$ and $\omega_{2}$, and firm 2 cannot distinguish between $\omega_{1}$ and $\omega_{3}$. In all states of

\footnotetext{
${ }^{12}$ This condition did not appear in the statement of Theorem 1C. It is needed only when prices are restricted to be non-negative. Indeed, without $\bar{Q}$ 's measurability with respect to both fields, there are counterexamples to uniqueness even if all firms have the same information, see Lagerlöf (2007).
} 
nature firms face the same quadratic inverse demand function

$$
P(Q)=\max \left\{1-Q^{2}, 0\right\}
$$

Thus, firms know the inverse demand in every state of nature, but have incomplete information about their costs. ${ }^{13}$ Firm 1 has a constant marginal cost of $\frac{1}{100}$ in states $\omega_{1}$ and $\omega_{2}$, while its marginal cost is 2 in $\omega_{3}$. Firm 2 has a constant marginal cost of $\frac{1}{100}$ in states $\omega_{1}$ and $\omega_{3}$, while its marginal cost is 2 in $\omega_{2}$.

Since in $\omega_{3}$ the marginal revenue of firm 1 is always below its marginal cost, firm 1 produces zero in this state in any best response. Similarly, firm 2 produces zero in $\omega_{2}$ in any best response. It follows that each firm $i$ 's strategy $q^{i}$ can, without loss of generality, be identified with a scalar: $q^{1}$ can be viewed as the quantity $x$ produced by firm 1 in state $\omega_{1}$ (and thus also in $\omega_{2}$ ), and $q^{2}$ as the quantity $y$ produced by firm 2 in state $\omega_{1}$ (and thus also in $\omega_{3}$ ).

We claim that both

$$
q_{*}=\left(x_{*}, y_{*}\right)=\left(\frac{3}{10} \sqrt{2}, \frac{3}{10} \sqrt{2}\right) \approx(0.42426,0.42426)
$$

and

$$
q_{* *}=\left(x_{* *}, y_{* *}\right)=\left(\frac{7}{30} \sqrt{6}, \frac{7}{30} \sqrt{6}\right) \approx(0.57155,0.57155)
$$

are Cournot equilibria.

Let us show first that $q_{*}$ is a Cournot equilibrium. For $y \in\left[0,1-x_{*}\right]$ the expected profit of firm 2 ,

$$
\begin{aligned}
U^{2}\left(x_{*}, y\right) & =\frac{1}{3} u^{2}\left(\omega_{1},\left(x_{*}, y\right)\right)+\frac{1}{3} u^{2}\left(\omega_{2},\left(x_{*}, 0\right)\right)+\frac{1}{3} u^{2}\left(\omega_{3},(0, y)\right) \\
& =\frac{1}{3} y\left(1-\left(\frac{3}{10} \sqrt{2}+y\right)^{2}\right)+\frac{1}{3} y\left(1-y^{2}\right)-\frac{2}{3} \frac{y}{100}
\end{aligned}
$$

has a (unique) maximum on $\left[0,1-x_{*}\right]$ at $y=y_{*}=\frac{3}{10} \sqrt{2}$. Thus firm 2 has no incentive to deviate from $y_{*}$ to another strategy in $\left[0,1-x_{*}\right]$. Now, for $y \in\left[1-x_{*}, 1\right]$,

$$
U^{2}\left(x_{*}, y\right)=\frac{1}{3} y\left(1-y^{2}\right)-\frac{2}{3} \frac{y}{100} .
$$

\footnotetext{
${ }^{13}$ Lagerlöf (2007) provides an example of equilibrium non-uniqueness with symmetrically informed firms but with incomplete information on the inverse demand. This example shows that knowing the inverse demand does not guarantee uniqueness either.
} 
The maximum of $\frac{1}{3} y\left(1-y^{2}\right)-\frac{2}{3} \frac{y}{100}$ on $\left[1-x_{*}, 1\right]$ is attained at $y=\frac{7}{30} \sqrt{6} \approx 0.57155$. This maximum is equal to $\frac{343}{6750} \sqrt{6} \approx 0.12447$, and therefore firm 2 has no incentive to deviate from $y_{*}$ (that gives it a payoff $\left.U^{2}\left(x_{*}, y_{*}\right) \approx 0.15274\right)$ to a strategy in $\left[1-x_{*}, 1\right]$. Since producing more than 1 would yield a negative expected profit, we have shown that firm 2 will not deviate unilaterally from $q_{*}$. By symmetry, the same holds for firm 1 , and thus $q_{*}$ is indeed a Cournot equilibrium.

We show next that $q_{* *}$ is a Cournot equilibrium. For $y \in\left[1-x_{* *}, 1\right]$ the expected profit of firm 2 ,

$$
U^{2}\left(x_{* *}, y\right)=\frac{1}{3} y\left(1-y^{2}\right)-\frac{2}{3} \frac{y}{100},
$$

reaches the maximum value of $\frac{343}{6750} \sqrt{6} \approx 0.12447$ at $y=y_{* *}=\frac{7}{30} \sqrt{6}$. Thus, firm 2 has no incentive to deviate from $y_{* *}$ to another strategy in $\left[1-x_{* *}, 1\right]$. For $y \in\left[0,1-x_{* *}\right]$, the expected profit of firm 2

$$
U^{2}\left(x_{* *}, y\right)=\frac{1}{3} y\left(1-\left(\frac{7}{30} \sqrt{6}+y\right)^{2}\right)+\frac{1}{3} y\left(1-y^{2}\right)-\frac{2}{3} \frac{y}{100},
$$

reaches the maximum value of $\approx 0.11798$ at $y \approx 0.36792$. Hence firm 2 has no incentive to deviate from $y_{* *}$ to a strategy in $\left[0,1-x_{* *}\right]$. Since producing more than 1 would yield negative expected profit, this shows that firm 2 will not deviate unilaterally from $q_{* *}$. By symmetry, the same holds for firm 1, and thus $q_{* *}$ is another Cournot equilibrium of the duopoly.

Remark 2 (Infinitely many states of nature). Throughout this paper we maintained the assumption that the set of states of nature $\Omega$ is finite. However, this assumption is by no means necessary, and was made only to simplify the presentation. In Einy et al (2007), a discussion paper on which this article is based, the uncertainty is represented by a probability space $(\Omega, \digamma, \mu)$, where $\Omega$ is a (possibly infinite) set of states of nature, $\digamma$ is a $\sigma$-field of subsets of $\Omega$, and $\mu$ is a common prior. Firm $i$ 's information is described by a $\sigma$-subfield $\digamma^{i}$ of $\digamma$, which is not necessarily generated by a partition of $\Omega$. The results on existence and uniqueness of Cournot equilibrium remain valid in this more general context. Their proofs follow very closely those presented here, but some additional assumptions are made, which are not needed when $\Omega$ is finite. In particular, it is assumed that the demand intercept $\bar{Q}$ is bounded, and that the state-dependent inverse demand function, cost 
functions, and their first and second order derivatives, are bounded uniformly in $\omega$ on some sufficiently big interval $[0, M]$. 


\section{References}

1. Amir, R. (1996). "Cournot Oligopoly and the Theory of Supermodular Games," Games and Economic Behavior 15, pp. 132-148.

2. Billingsley, P. (1995). Probability and Measure (third edition). John Wiley and Sons, New York.

3. Einy, E., Moreno, D. and B. Shitovitz (2002). "Information Advantage in Cournot Oligopoly," Journal of Economic Theory 106, pp. 151-160.

4. Einy, E., Moreno, D. and B. Shitovitz (2003). "The Value of Public Information in a Cournot Duopoly," Games and Economic Behavior 44, pp. 272-285.

5. Einy, E., Haimanko, O, Moreno, D. and B. Shitovitz (2007). "On the Existence of Bayesian Cournot Equilibrium," Working Paper 07-06 in Economic Series 03, Departamento de Economia, Universidad Carlos III de Madrid.

6. Gal-Or, E. (1985). "Information Sharing in Oligopoly," Econometrica 53, pp. $85-92$.

7. Gal-Or, E. (1986). "Information Transmission - Cournot and Bertrand Equilibria," Review of Economic Studies 53, pp. 329-344.

8. Hon-Snir, S., B. Shitovitz and M. Spiegel (2007). "Bayesian Equilibrium in a Public Good Economy," manuscript.

9. Kukushkin, N. S. (1994). "A Fixed-point Theorem for Decreasing Mappings," Economics Letters 46, pp. 23-26.

10. Lagerlöf, J. (2006). "Equilibrium Uniqueness in a Cournot Model with Demand Uncertainty," Topics in Theoretical Economics 6, Article 19.

11. Lagerlöf, J. (2007). "Insisting on a Non-Negative Price: Oligopoly, Uncertainty, Welfare, and Multiple Equilibria," International Journal of Industrial Organization, forthcoming. 
12. Malueg, D. A. and S. O. Tsutsui (1998). "Oligopoly Information Exchange When Non-Negative Price and Output Constraints May Bind," Australian Economic Papers 37, pp. 363-371.

13. Milgrom, P. and J. Roberts (1990). "Rationalizability, Learning, and Equilibrium in Games with Strategic Complementarities," Econometrica 58, pp. 1255-1277.

14. Novshek, W. (1985). "On the Existence of Cournot Equilibrium," Review of Economic Studies 52, pp. 85-98.

15. Raith, M. (1996). "A General Model of Information Sharing in Oligopoly," Journal of Economic Theory 71, pp. 260-288.

16. Sakai, Y. (1985). "The Value of Information in a Simple Duopoly Model," Journal of Economic Theory 36, pp. 36-54.

17. Sakai, Y. (1986). "Cournot and Bertrand Equilibria under Imperfect Information," Journal of Economics (Zeitschrift fur Nationalokonomie) 46, pp. 213232.

18. Shapiro, C. (1986). "Exchange of Cost Information in Oligopoly," Review of Economic Studies 53, pp. 213-232.

19. Szidarovszky, F. and S. Yakowitz (1977). "A New Proof of Existence and Uniqueness of the Cournot Equilibrium," International Economic Review 18.

20. Vives, X. (1984). "Duopoly Informaton Equilibrium: Cournot and Bertrand," Journal of Economic Theory 34, pp. 71-94.

21. Vives, X. (1988). "Aggregation of Information in Large Cournot Markets," Econometrica 56, pp. 851-876.

22. Vives, X. (1990). "Nash Equilibrium with Strategic Complementarities," Journal of Mathematical Economics 19, pp. 305-321.

23. Vives, X. (1999). Oligopoly Pricing: Old Ideas and New Tools. The MIT Press, Cambridge, Massachusetts. 


\section{Appendix}

Denote by $B(\Omega)$ the set of all non-negative real-valued functions on $\Omega$. The following definition of a partial order on $B(\Omega)$ will be needed in the sequel: if $g, h \in B(\Omega)$, $g \geq h$ (respectively, $g>h$ ) if and only if $g(\omega) \geq h(\omega)$ (respectively, $g(\omega)>h(\omega)$ ) for every $\omega \in \Omega$. Similarly, we will say that $g \geq h$ (respectively, $g>h$ ) on $A \subset \Omega$ if and only if $g(\omega) \geq h(\omega)$ (respectively, $g(\omega)>h(\omega)$ ) for every $\omega \in A$.

\subsection{Proofs of theorem $1 \mathrm{~A}$ and $1 \mathrm{~B}$}

\subsubsection{Part I: Proof of theorem 1A}

We will show first that for each $\omega \in \Omega$ the profit function $u_{\omega}^{1}(\cdot) \equiv u^{1}(\omega, \cdot)$ of firm 1 has decreasing differences in the first coordinate, that is, if $x_{1} \geq x_{2} \geq 0$ and $y_{1} \geq y_{2} \geq 0$, then

$$
\left[u_{\omega}^{1}\left(x_{1}, y_{2}\right)-u_{\omega}^{1}\left(x_{2}, y_{2}\right)\right]-\left[u_{\omega}^{1}\left(x_{1}, y_{1}\right)-u_{\omega}^{1}\left(x_{2}, y_{1}\right)\right] \geq 0
$$

i.e.,

$$
\left[x_{1} P\left(\omega, x_{1}+y_{2}\right)-x_{2} P\left(\omega, x_{2}+y_{2}\right)\right]-\left[x_{1} P\left(\omega, x_{1}+y_{1}\right)-x_{2} P\left(\omega, x_{2}+y_{1}\right)\right] \geq 0 .
$$

Since $P(\omega, \cdot)$ is continuously differentiable, this condition is equivalent to

$$
\frac{\partial}{\partial y_{2}}\left[x_{1} P\left(\omega, x_{1}+y_{2}\right)-x_{2} P\left(\omega, x_{2}+y_{2}\right)\right] \leq 0
$$

or

$$
x_{1} P^{\prime}\left(\omega, x_{1}+y_{2}\right)-x_{2} P^{\prime}\left(\omega, x_{2}+y_{2}\right) \leq 0,
$$

for every $x_{1} \geq x_{2} \geq 0$ and $y_{2} \geq 0$. This condition, in turn, is equivalent (since $P^{\prime}(\omega, \cdot)$ is also continuously differentiable by condition $A$ ) to

$$
\frac{\partial}{\partial x_{2}}\left[x_{2} P^{\prime}\left(\omega, x_{2}+y_{2}\right)\right] \leq 0
$$

or

$$
x_{2} P^{\prime \prime}\left(\omega, x_{2}+y_{2}\right)+P^{\prime}\left(\omega, x_{2}+y_{2}\right) \leq 0 \text {, }
$$

for every $x_{2} \geq 0$ and $y_{2} \geq 0$. However, (16) is implied by assumption (ii) and condition $A$ on $P$. 
From (15) it follows that the expected profit function $U^{1}$ of firm 1 also has decreasing differences in the first coordinate: for every $\left(q^{1}, q^{2}\right),\left(\widetilde{q}^{1}, \widetilde{q}^{2}\right) \in B\left(\Omega, \Pi^{1}\right) \times$ $B\left(\Omega, \Pi^{2}\right)$ such that $q^{1} \geq \widetilde{q}^{1}, q^{2} \geq \widetilde{q}^{2}$,

$$
\left[U^{1}\left(q^{1}, \widetilde{q}^{2}\right)-U^{1}\left(\widetilde{q}^{1}, \widetilde{q}^{2}\right)\right]-\left[U^{1}\left(q^{1}, q^{2}\right)-U^{1}\left(\widetilde{q}^{1}, q^{2}\right)\right] \geq 0
$$

Similarly, the expected payoff function $U^{2}$ of firm 2 has decreasing differences in the second coordinate.

With the partial order $\geq$ on $B\left(\Omega, \Pi^{i}\right)$ and the pointwise convergence topology on it, for every $g, h \in B\left(\Omega, \Pi^{i}\right)$ with $g \geq h$ the interval $[g, h] \subset B\left(\Omega, \Pi^{i}\right)$ is a compact lattice. Now denote the constant function on $\Omega$ which is fixed at the level ${ }^{14} Z$ (respectively, 0) by the same symbol, $Z$ (respectively, 0), and let strategy profiles of the firms be restricted to $S^{1} \times S^{2} \equiv[0, Z] \times[0, Z]$, a product of compact lattices. Since the state-dependent inverse demand and cost functions are continuous (by assumption (i) and condition $A$ ), then each function $U^{i}$ is continuous on $S^{1} \times S^{2}$ in both coordinates.

Now reverse the order in $S^{2}$, i.e., replace the order " $\geq$ " with " $\geq$ " according to which $g \geq ' h$ if and only if $h \geq g$. Then both $U^{1}$ and $U^{2}$ exhibit increasing, rather than decreasing, differences. The reversal of order has no effect on continuity of $U^{1}$ and $U^{2}$. Since both $S^{1}$ and $S^{2}$ are compact lattices, theorem 5 of Milgrom and Roberts (1990) implies that there exists a Cournot equilibrium when strategy profiles of the firms are restricted to be in $S^{1} \times S^{2} .{ }^{15}$ Denote one such equilibrium by $\left(q_{*}^{1}, q_{*}^{2}\right)$. If $\left(q^{1}, q^{2}\right) \in B\left(\Omega, \Pi^{1}\right) \times B\left(\Omega, \Pi^{2}\right)$, denote by $r^{i}$ the strategy of $i$ which is equal to 0 on the $\Pi^{i}$-measurable set $A=\left\{\omega \mid q^{i}(\omega)>\bar{q}^{i}(\omega)\right\}$, and to $q^{i}$ on $A^{c}$, for $i=1,2$. Notice that $U^{1}\left(q^{1}, q_{*}^{2}\right) \leq U^{1}\left(r^{1}, q_{*}^{2}\right)$ and $U^{2}\left(q_{*}^{1}, q^{2}\right) \leq U^{2}\left(q_{*}^{1}, r^{2}\right)$, as follows from assumptions (i) and (iii). Therefore

$$
U^{1}\left(q_{*}^{1}, q_{*}^{2}\right) \geq U^{1}\left(r^{1}, q_{*}^{2}\right) \geq U^{1}\left(q^{1}, q_{*}^{2}\right)
$$

and

\footnotetext{
${ }^{14}$ Recall that $Z$ is a level of output above which each firm's monopoly profit is negative, in every state of nature.

${ }^{15}$ One more thing needs to be verified before applying this theorem, namely that $U^{1}$ is supermodular in $q^{1}$ for fixed $q^{2}$, i.e., for every $q^{1}, \widetilde{q}^{1} \in S^{1}$ and $q^{2} \in S^{2}, U^{1}\left(q^{1}, q^{2}\right)+U^{1}\left(\widetilde{q}^{1}, q^{2}\right) \leq$ $U^{1}\left(\max \left(q^{1}, \widetilde{q}^{1}\right), q^{2}\right)+U^{1}\left(\min \left(q^{1}, \widetilde{q}^{1}\right), q^{2}\right)$, and similarly for $U^{2}$. However, it can be easily checked that this inequality actually holds as equality.
} 


$$
U^{2}\left(q_{*}^{1}, q_{*}^{2}\right) \geq U^{2}\left(q_{*}^{1}, r^{2}\right) \geq U^{2}\left(q_{*}^{1}, q^{2}\right)
$$

since $\left(q_{*}^{1}, q_{*}^{2}\right)$ is a Cournot equilibrium when the strategy profiles of the firms are restricted to $S^{1} \times S^{2}$. But these inequalities show that $\left(q_{*}^{1}, q_{*}^{2}\right)$ is actually a Cournot equilibrium without any restrictions on strategies.

\subsubsection{Part II: Proof of theorem 1B}

The proof is a direct consequence of the Nash existence theorem. First, for each $\omega \in \Omega, u_{\omega}^{i}(\cdot) \equiv u^{i}(\omega, \cdot)$ is concave in strategies of firm $i$. Indeed, the second derivative of $q^{i}(\omega) P(\omega, Q(\omega))$ with respect to $q^{i}(\omega)$ is equal to $q^{i}(\omega) P^{\prime \prime}(\omega, Q(\omega))+$ $2 P^{\prime}(\omega, Q(\omega))$, which is non-positive as follows from assumption (ii) and condition $B$. Thus, $q^{i}(\omega) P(\omega, Q(\omega))$ is concave in $q^{i}(\omega)$, and from convexity of $c^{i}(\omega, \cdot)$ it follows that $u_{\omega}^{i}(q(\omega))=q^{i}(\omega) P(\omega, Q(\omega))-c^{i}\left(\omega, q^{i}(\omega)\right)$ is also concave in $q^{i}(\omega)$. The expected profit function $U^{i}$ clearly inherits concavity in $q^{i}$ from $u_{\omega}^{i}$.

Second, following notations of Part I, restrict the strategy set of each firm $i$ to the compact $S^{i}=[0, Z]$. As in Part I, $U^{i}$ is continuous in all coordinates simultaneously on the compact cube $[0, Z]^{N}$. Thus, all ingredients for the existence of Nash equilibrium are in place, with the above restriction of strategies. However, the restricted equilibrium is an equilibrium in the unrestricted oligopoly as well, which can be shown again exactly as in Part I.

\subsection{Proof of theorem 1C}

Since all conditions of theorem 1B are satisfied, the oligopoly has at least one Cournot equilibrium. We will show that it is unique.

Let $q_{*}$ be a Cournot equilibrium, and pick a firm $i$. Since

$$
E\left(q^{i}(\cdot) P\left(\cdot, \sum_{j \neq i} q_{*}^{j}(\cdot)+q^{i}(\cdot)\right)-c^{i}\left(\cdot, q^{i}(\cdot)\right) \mid \Pi^{i}(\omega)\right)
$$

is maximized (and in particular locally maximized) at $q^{i}=q_{*}^{i}$ for every $\omega \in \Omega$, the Kuhn-Tucker conditions are satisfied:

$$
E\left(q_{*}^{i}(\cdot) P^{\prime}\left(\cdot, Q_{*}(\cdot)\right)+P\left(\cdot, Q_{*}(\cdot)\right)-\left(c^{i}\right)^{\prime}\left(\cdot, q_{*}^{i}(\cdot)\right) \mid \Pi^{i}(\omega)\right)=0
$$


for every $\omega$ in which $q_{*}^{i}>0$, and

$$
E\left(q_{*}^{i}(\cdot) P^{\prime}\left(\cdot, Q_{*}(\cdot)\right)+P\left(\cdot, Q_{*}(\cdot)\right)-\left(c^{i}\right)^{\prime}\left(\cdot, q_{*}^{i}(\cdot)\right) \mid \Pi^{i}(\omega)\right) \leq 0
$$

for every $\omega$ in which $q_{*}^{i}=0$.

Note that for each $\omega \in \Omega$ the function

$$
F(q, Q)=q P^{\prime}(\omega, Q)+P(\omega, Q)-\left(c^{i}\right)^{\prime}(\omega, q)
$$

is decreasing in $q$ and non-increasing in $Q$ when $q \leq Q$. Indeed, $\frac{\partial F}{\partial q}=P^{\prime}(\omega, Q)-$ $\left(c^{i}\right)^{\prime \prime}(\omega, q)<0$ since $P$ is strictly decreasing and $c$ is convex by assumption, and $\frac{\partial F}{\partial Q}=q P^{\prime \prime}(\omega, Q)+P^{\prime}(\omega, Q) \leq 0$ as follows from $P(\omega, \cdot)$ being decreasing and condition $A$. Now suppose that $q_{*}$ and $q_{* *}$ are two Cournot equilibria. That $F$ is decreasing in $q$ and non-increasing in $Q$ implies that one cannot have

$$
\left(q_{*}^{i}, Q_{*}\right)<\left(q_{* *}^{i}, Q_{* *}\right) \text { or }\left(q_{*}^{i}, Q_{*}\right)>\left(q_{* *}^{i}, Q_{* *}\right)
$$

(inequality in both coordinates and strict inequality in the first coordinate) on any atom $\Pi^{i}(\omega)$ of $\Pi^{i}$. This is because otherwise conditions (18) and (19) would not hold simultaneously for $\max \left(\left(q_{*}^{i}, Q_{*}\right),\left(q_{* *}^{i}, Q_{* *}\right)\right)$. To summarize, any firm's equilibrium strategy and the aggregate output in equilibrium cannot move in the same direction:

$$
\left(q_{*}^{i}, Q_{*}\right) \nless\left(q_{* *}^{i}, Q_{* *}\right) \text { and }\left(q_{*}^{i}, Q_{*}\right) \ngtr\left(q_{* *}^{i}, Q_{* *}\right)
$$

on any element of $\Pi^{i}$.

We will next show that every Cournot equilibrium satisfies the equal treatment property, i.e., that strategies of firms of the same type are equal. Indeed, if $q_{*}$ is a Cournot equilibrium, and $q_{*}^{i} \neq q_{*}^{j}$ where $i$ and $j$ are firms of the same type, then consider an $n$-tuple $q_{* *}$ obtained from $q_{*}$ by interchanging $i$ and $j$. Clearly, $q_{* *}$ is also a Cournot equilibrium. However, if $\Pi^{i}(\omega) \in \Pi^{i}$ is a set on which w.l.o.g. $q_{*}^{i}>q_{*}^{j}=q_{* *}^{i}$, then the obvious fact that $Q_{*}=Q_{* *}$ leads to contradiction with (20). Thus, the equal treatment property holds in any Cournot equilibrium.

Now suppose that $q_{*}$ and $q_{* *}$ are Cournot equilibria in the oligopoly. We will show that they coincide. Due to the equal treatment property, $Q_{*}(\omega)=|K| q_{*}^{1}(\omega)+$ $|M| q_{*}^{2}(\omega)$, and it will suffice to establish that $q_{*}^{i}=q_{* *}^{i}$ for $i=1,2$. If $q_{*}^{2}$ and $q_{* *}^{2}$ are not equal everywhere, then there exists $\omega \in \Omega$ such that w.l.o.g.

$$
q_{*}^{2}>q_{* *}^{2} \text { on } \Pi^{2}(\omega)
$$


Consequently,

$$
q_{*}^{1} \leq q_{* *}^{1} \text { on } \Pi^{2}(\omega)
$$

Indeed, if (22) does not hold, there is $\omega^{\prime} \in \Pi^{2}(\omega)$ with $q_{*}^{1}>q_{* *}^{1}$ on $\Pi^{1}\left(\omega^{\prime}\right)$. But $\Pi^{1}\left(\omega^{\prime}\right) \subset \Pi^{2}(\omega)$ since the information partition of 1 is finer than that of 2 . Thus, from (21), also $Q_{*}>Q_{* *}$ on $\Pi^{1}\left(\omega^{\prime}\right)$, contradicting (20)).

We now claim that

$$
Q_{*} \geq Q_{* *} \text { on } \Pi^{2}(\omega)
$$

Indeed, both $Q_{*}$ and $Q_{* *}$ are measurable with respect to the information partition of the more informed firm 1 , and thus, if (23) does not hold, there is $\omega^{\prime \prime} \in \Pi^{2}(\omega)$ with

$$
Q_{*}<Q_{* *} \text { on } \Pi^{1}\left(\omega^{\prime \prime}\right)
$$

Strict inequality in $(22)$ on $\Pi^{1}\left(\omega^{\prime \prime}\right) \subset \Pi^{2}(\omega)$ together with (24) would contradict (20), and thus $q_{*}^{1}=q_{* *}^{1}$ on $\Pi^{1}\left(\omega^{\prime \prime}\right)$. But then $Q_{*}>Q_{* *}$ on $\Pi^{1}\left(\omega^{\prime \prime}\right)$ because of $(21)$, contrary to the choice of $\omega^{\prime \prime}$. Thus (23) must hold.

But now (21) and (23) contradict (20). Thus, strategies $q_{*}^{2}$ and $q_{* *}^{2}$ must coincide almost everywhere. Now, if $q_{*}^{1}$ differs from $q_{* *}^{1}$ on $\Pi^{1}(\omega)$ for some $\omega \in \Omega$, and w.l.o.g. $q_{*}^{1}>q_{* *}^{1}$ on $\Pi^{1}(\omega)$, then $Q_{*}>Q_{* *}$ on $\Pi^{1}(\omega)$ since $q_{*}^{2}=q_{* *}^{2}$, contradicting (20) again. We conclude that $q_{*}^{1}=q_{* *}^{1}$ as well.

\subsection{Proof of theorems $2 \mathrm{~A}$ and $2 \mathrm{~B}$}

First, restrict strategy sets of each firm $i$ to be $S^{i}=\left[0, \bar{q}^{i}\right]$. Note that for every strategy profile $q \in S^{1} \times \ldots \times S^{n}, Q \leq \sum_{i=1}^{n} \bar{q}^{i} \leq \bar{Q}$. Hence, strategy profiles in $S^{1} \times \ldots \times S^{n}$ have exactly the same properties as if conditions $A^{\prime}$ or $B^{\prime}$ held on the entire $\mathbb{R}_{+}$. Thus, just as in the proof of theorems $1 \mathrm{~A}$ and $1 \mathrm{~B}$ (replacing $S^{i}=[0, Z]$ with $\left.S^{i}=\left[0, \bar{q}^{i}\right]\right)$, there is a Cournot equilibrium $q_{*} \in S^{1} \times \ldots \times S^{n}$ in the oligopoly, provided all unilateral deviations of $i$ considered in (2) are in $S^{i}$.

To show that $q_{*}$ is a Cournot equilibrium in the unrestricted oligopoly as well, we now prove that unilateral deviations of $i$ to strategies outside $S^{i}$ are not profitable. Indeed, if $q^{i}$ is $i$ th strategy which is not in $S^{i}$, then for some $r^{i} \leq \bar{q}^{i}$, by (13)

$$
U^{i}\left(q_{*} \mid q^{i}\right) \leq U^{i}\left(q_{*} \mid r^{i}\right),
$$


and

$$
U^{i}\left(q_{*} \mid r^{i}\right) \leq U^{i}\left(q_{*}\right)
$$

since $r^{i} \in S^{i}$ and $q_{*}$ is a Cournot equilibrium when the strategy profiles of the firms are restricted to $S^{1} \times \ldots \times S^{n}$. This proves via (2) that $q_{*}$ is indeed a Cournot equilibrium of the oligopoly without restriction on strategies.

\subsection{Proof of Corollary 1}

Given a profile of strategies $q$, consider the strategy $r^{i}$ of $i$ which is equal to 0 on the $\Pi^{i}$-measurable set $A=\left\{\omega \mid q^{i}(\omega)>\bar{q}^{i}(\omega)\right\}$, and to $q^{i}$ on $A^{c}$. If $\omega \in A$,

$$
\begin{aligned}
E\left(u^{i}(\cdot, q(\cdot)) \mid \Pi^{i}(\omega)\right) & \leq E\left(u^{i}\left(\cdot,\left(q^{i}, 0^{-i}\right)\right) \mid \Pi^{i}(\omega)\right) \\
& \leq 0 \\
& =E\left(u^{i}\left(\cdot, q(\cdot) \mid r^{i}(\cdot)\right) \mid \Pi^{i}(\omega)\right)
\end{aligned}
$$

as follows from conditions (i), (ii), and (14). And if $\omega \in A^{c}$, then $q^{i}=r^{i}$ on $\Pi^{i}(\omega)$, and thus for every $\omega \in \Omega$

$$
E\left(u^{i}(\cdot, q(\cdot)) \mid \Pi^{i}(\omega)\right) \leq E\left(u^{i}\left(\cdot, q(\cdot) \mid r^{i}(\cdot)\right) \mid \Pi^{i}(\omega)\right) .
$$

By taking the expectation over $\omega$ in (25), we obtain (13). Existence of a Cournot equilibrium in a duopoly then follows from theorem $2 \mathrm{~A}$ under condition $A^{\prime}$, and in an oligopoly from theorem $2 \mathrm{~B}$ under condition $B^{\prime}$.

\subsection{Proof of theorem 2C}

Note that if $q_{*}$ is a Cournot equilibrium, then

$$
Q_{*}<\bar{Q}
$$

Indeed, if this is not case, consider an $\omega \in \Omega$ such that $Q_{*} \geq \bar{Q}$ on $\Pi^{1}(\omega)$ (such an $\omega$ exists since both $Q_{*}$ and $\bar{Q}$ are measurable with respect to the finest of all information partitions, $\left.\Pi^{1}\right)$. If there exists a firm $i \in K$ with $q_{*}^{i}>0$ on $\Pi^{1}(\omega)$, then $i$ would benefit by switching its output to zero on $\Pi^{1}(\omega)$ and saving its costs, contradicting (3). And if for all $i \in K q_{*}^{i}=0$ on $\Pi^{1}(\omega)$, then $\sum_{j \in M} q_{*}^{j}=Q_{*} \geq \bar{Q}$ on $\Pi^{1}(\omega)$. But since both $\sum_{j \in M} q_{*}^{j}$ and $\bar{Q}$ are measurable with respect to $\Pi^{2}$, there exists an $\omega^{\prime} \in \Omega$ 
such that $\Pi^{1}(\omega) \subset \Pi^{2}\left(\omega^{\prime}\right)$ and $\sum_{j \in M} q_{*}^{j} \geq \bar{Q}(>0)$ on $\Pi^{2}\left(\omega^{\prime}\right)$. Accordingly, there exists a firm $j \in M$ with $q_{*}^{j}>0$ on $\Pi^{2}\left(\omega^{\prime}\right)$, and just as before this means that $j$ has a profitable deviation from $q_{*}$ on $\Pi^{2}\left(\omega^{\prime}\right)$, contradicting (3). We conclude that (26) holds.

But if $q_{*}$ and $q_{* *}$ are two Cournot equilibria, it follows from (26) that $q_{*}, q_{* *}$, and all strategy profiles close to them ${ }^{16}$ have exactly the same properties as if the differentiability condition in $A^{\prime}$ held for all $Q \geq 0$ (i.e., as if $A^{\prime}$ had the original form $A$ ). We can therefore show that $q_{*}$ and $q_{* *}$ coincide, just as in the proof of theorem $1 \mathrm{C}$, using the first-order conditions derived from maximization of (17).

\footnotetext{
${ }^{16}$ What we have in mind are strategy profiles that constitute, at each state of nature, small unilateral deviations from $q_{*}$ or $q_{* *}$.
} 


$$
x=99 / 400
$$

Firm 2's Residual Demands

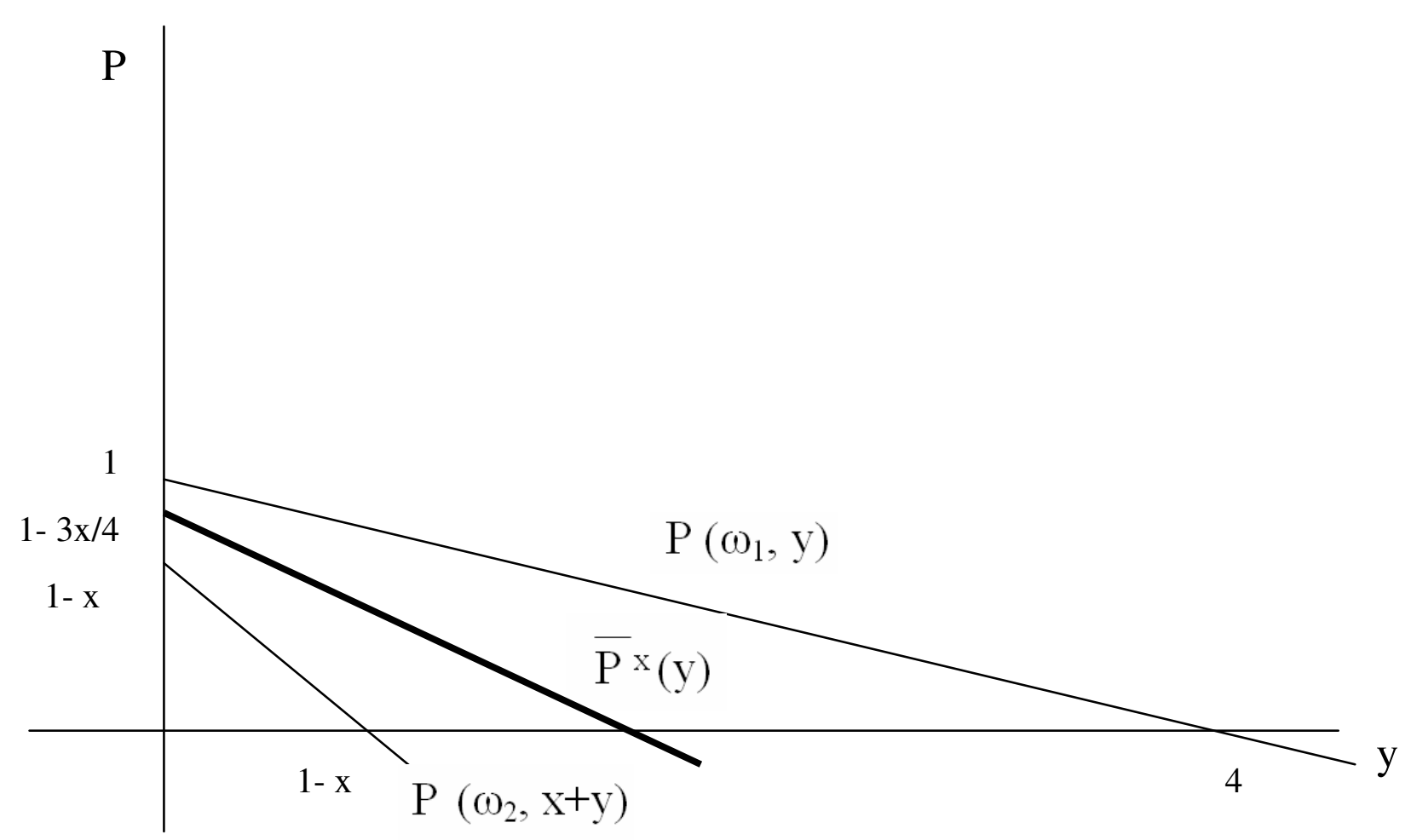

Figure 1a 
Firm 2's Payoff

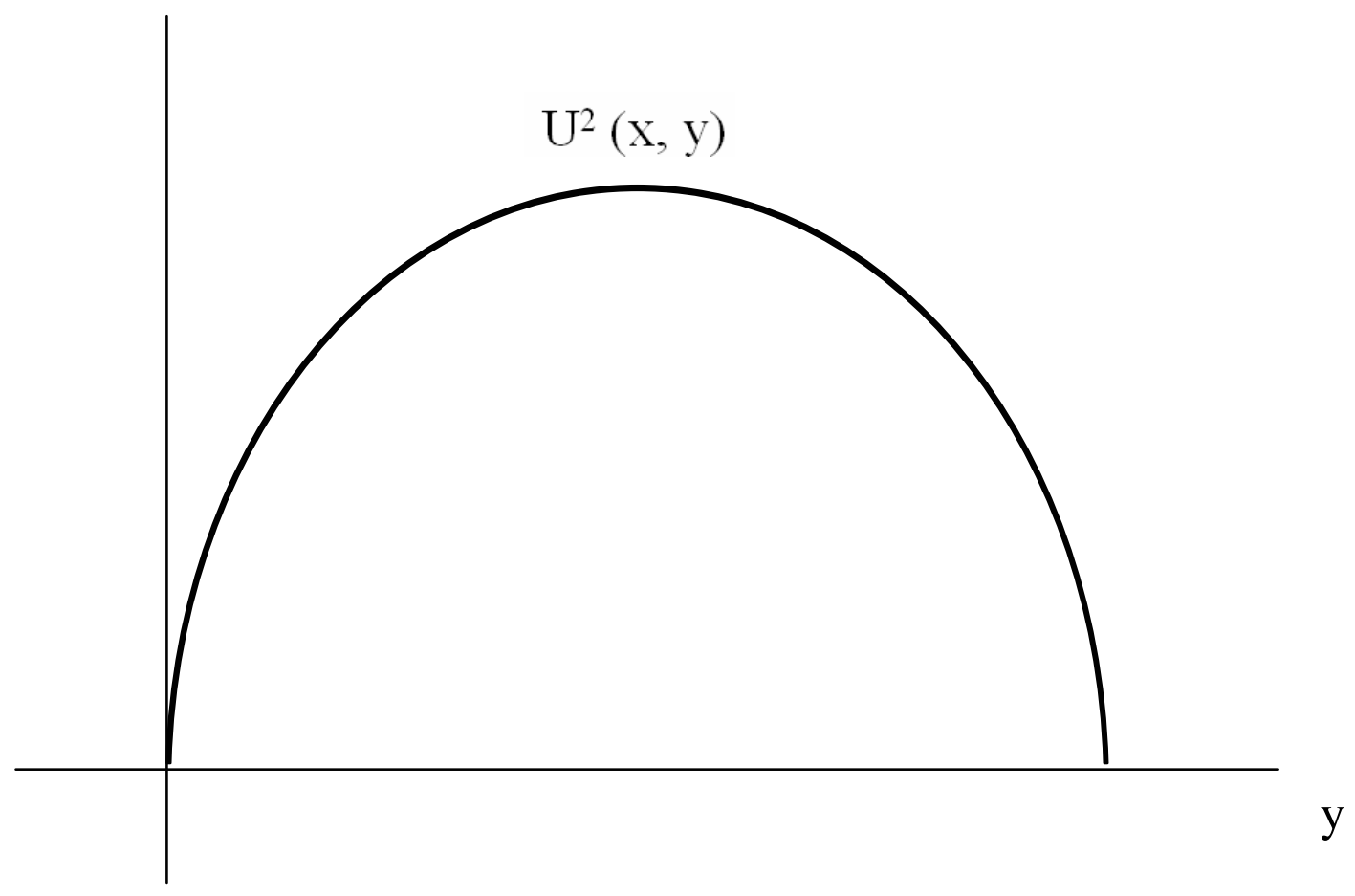

Figure 1b 
Firms' Reaction Functions

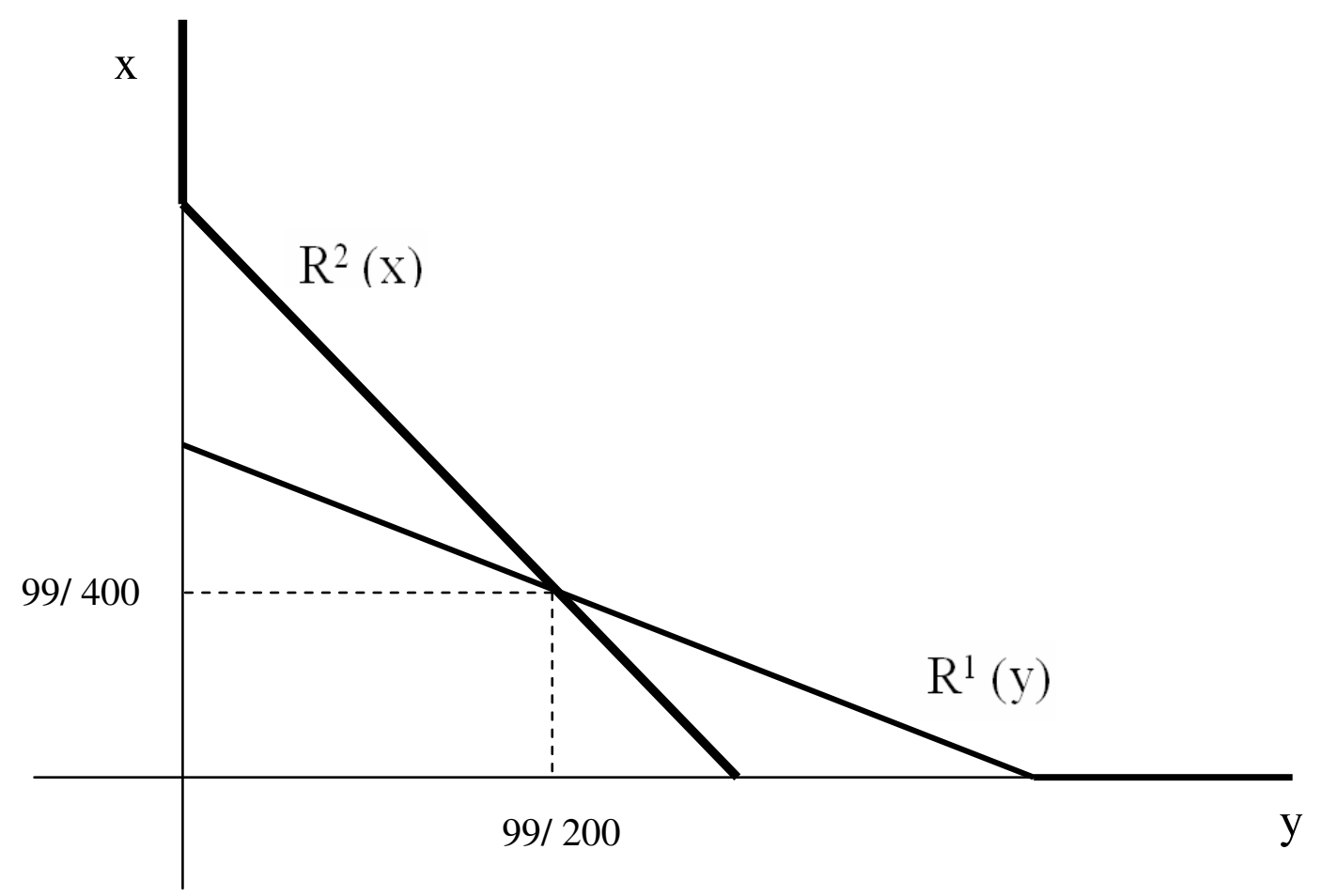

Figure 1c 


$$
x=99 / 400
$$

Firm 2's Residual Demands

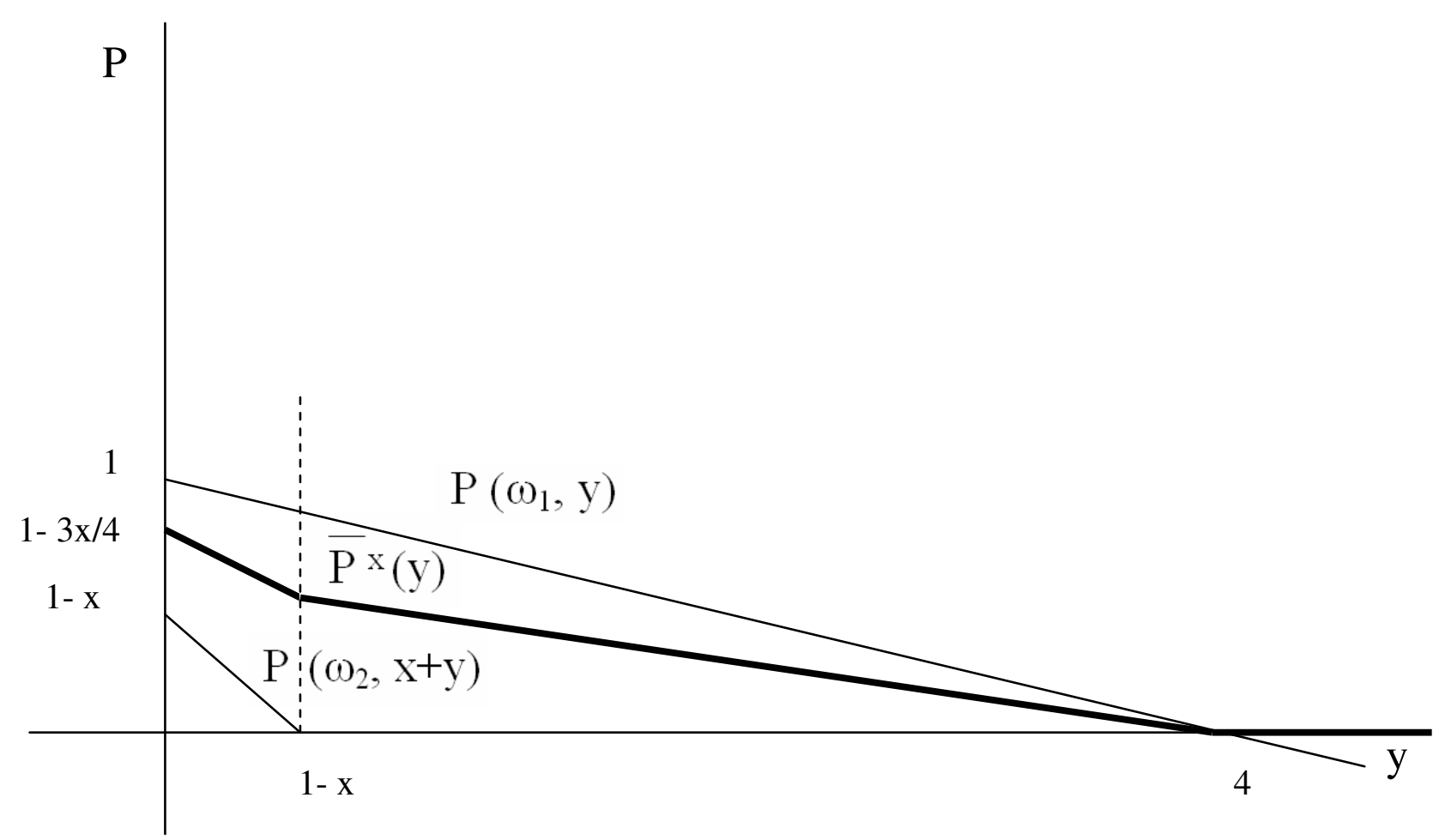

Figure 2a 
Firm 2's Payoff

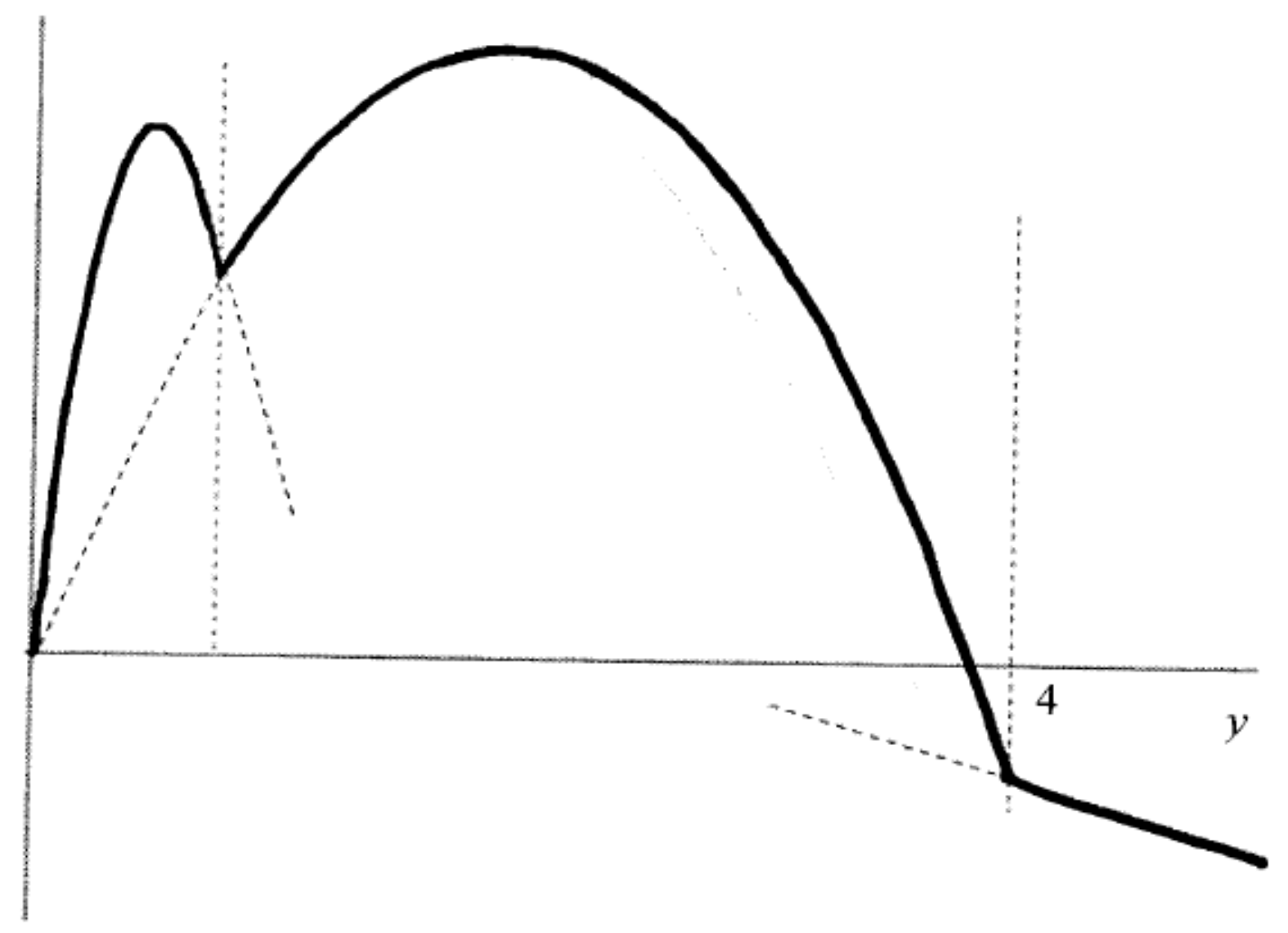

Figure 2b 
Firms' Reaction Functions

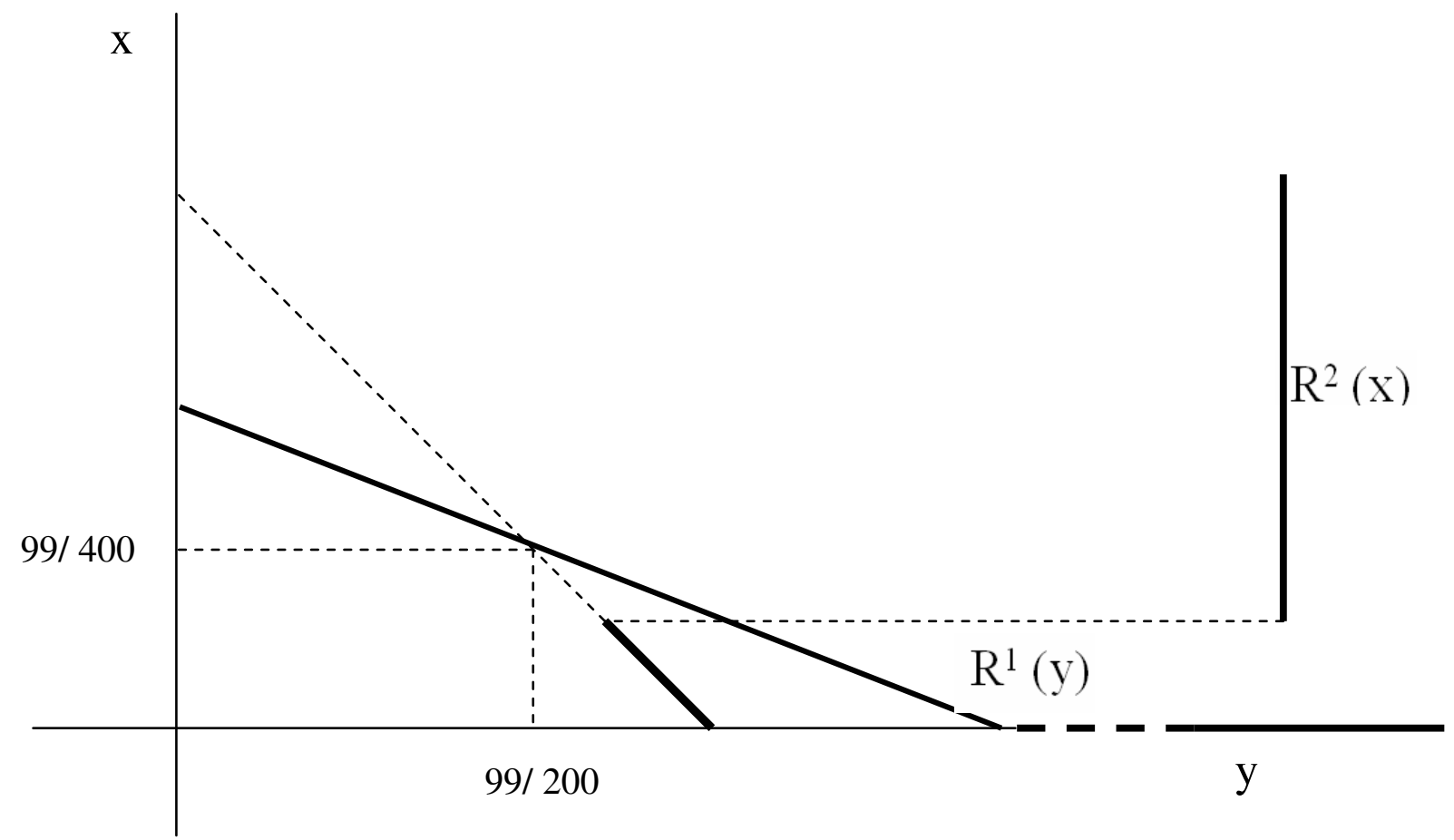

Figure 2c 


$$
\mathrm{x}=.22
$$

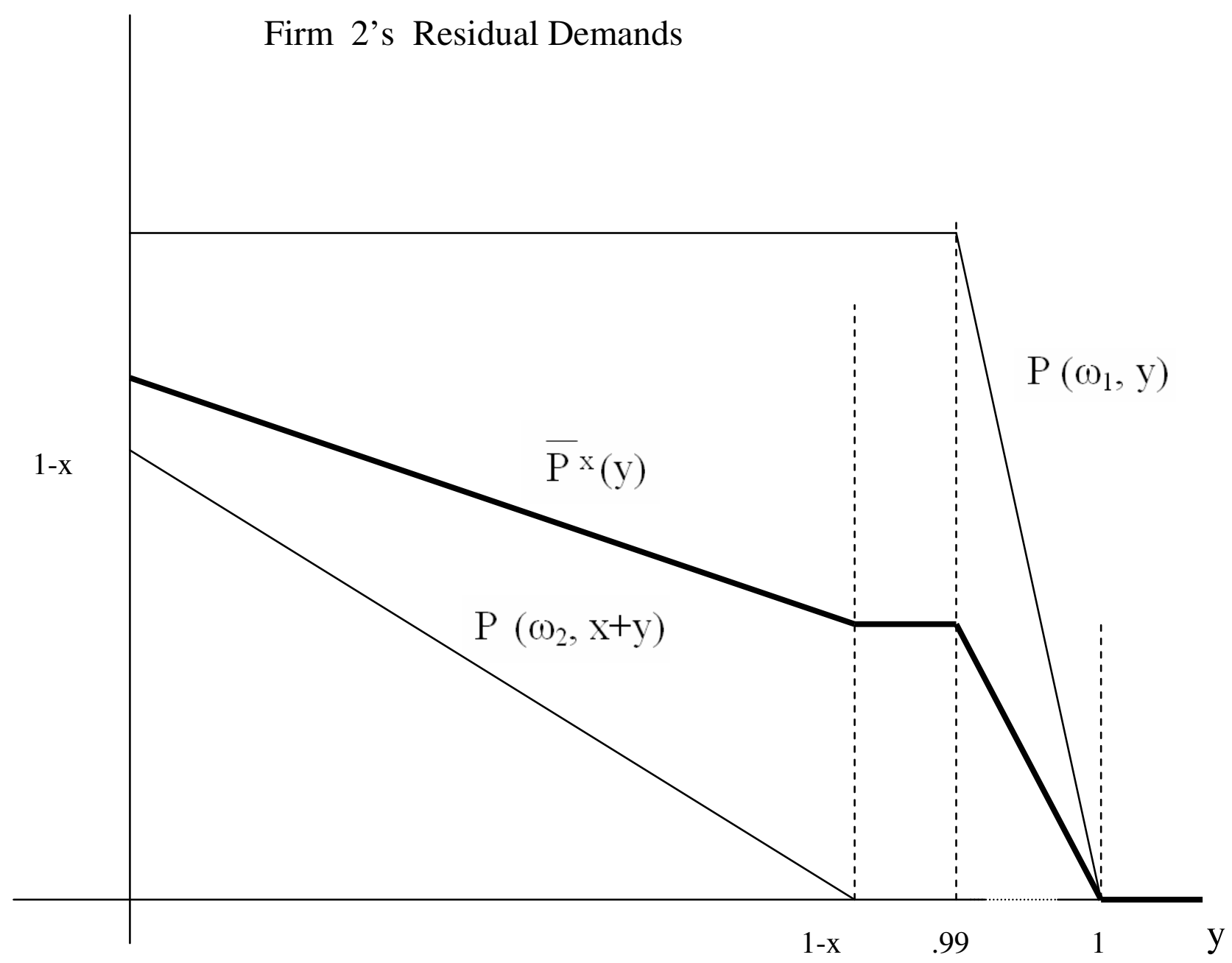

Figure 3a 
Firm 2's Payoff

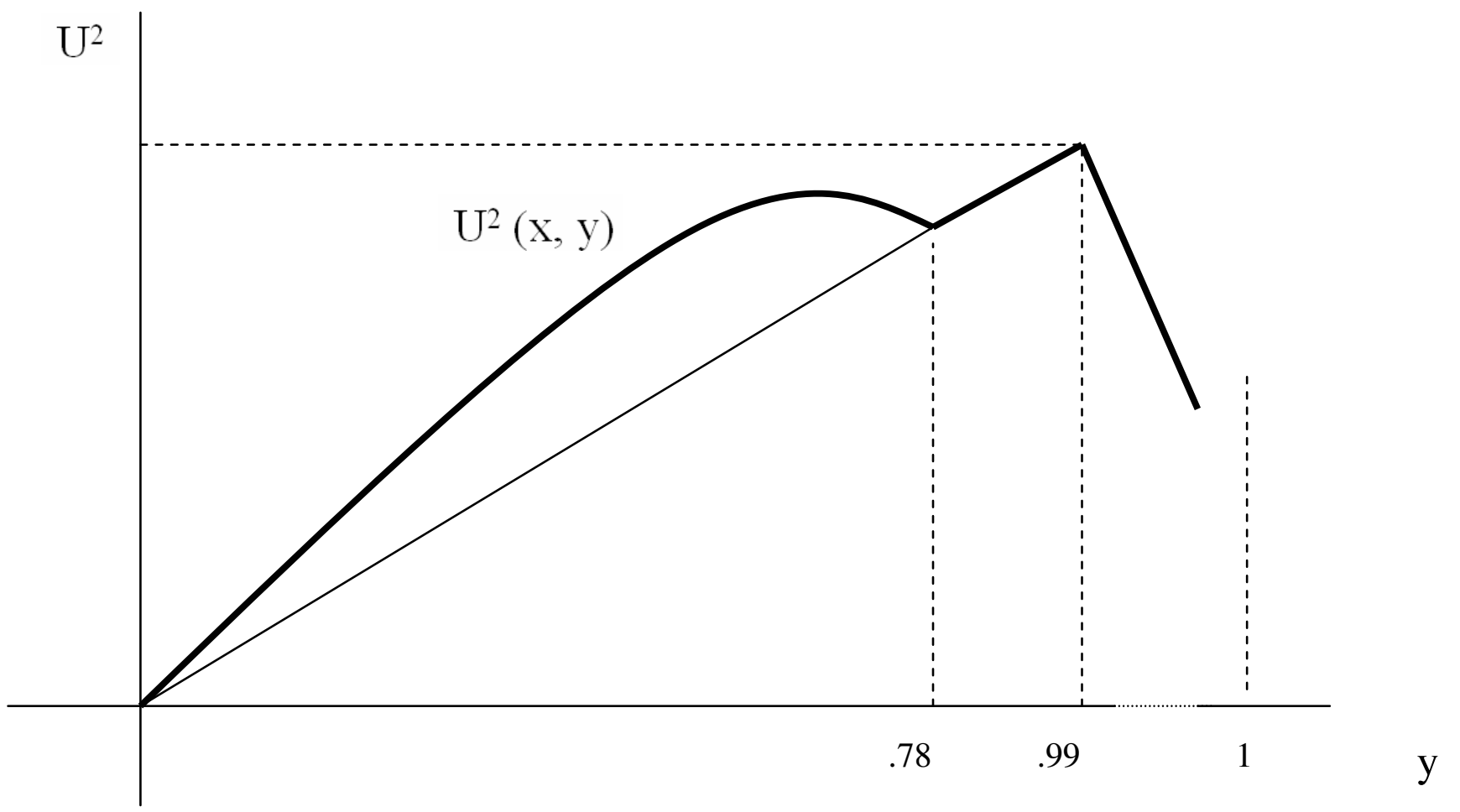

Figure 3b 
Firms' Reaction Functions

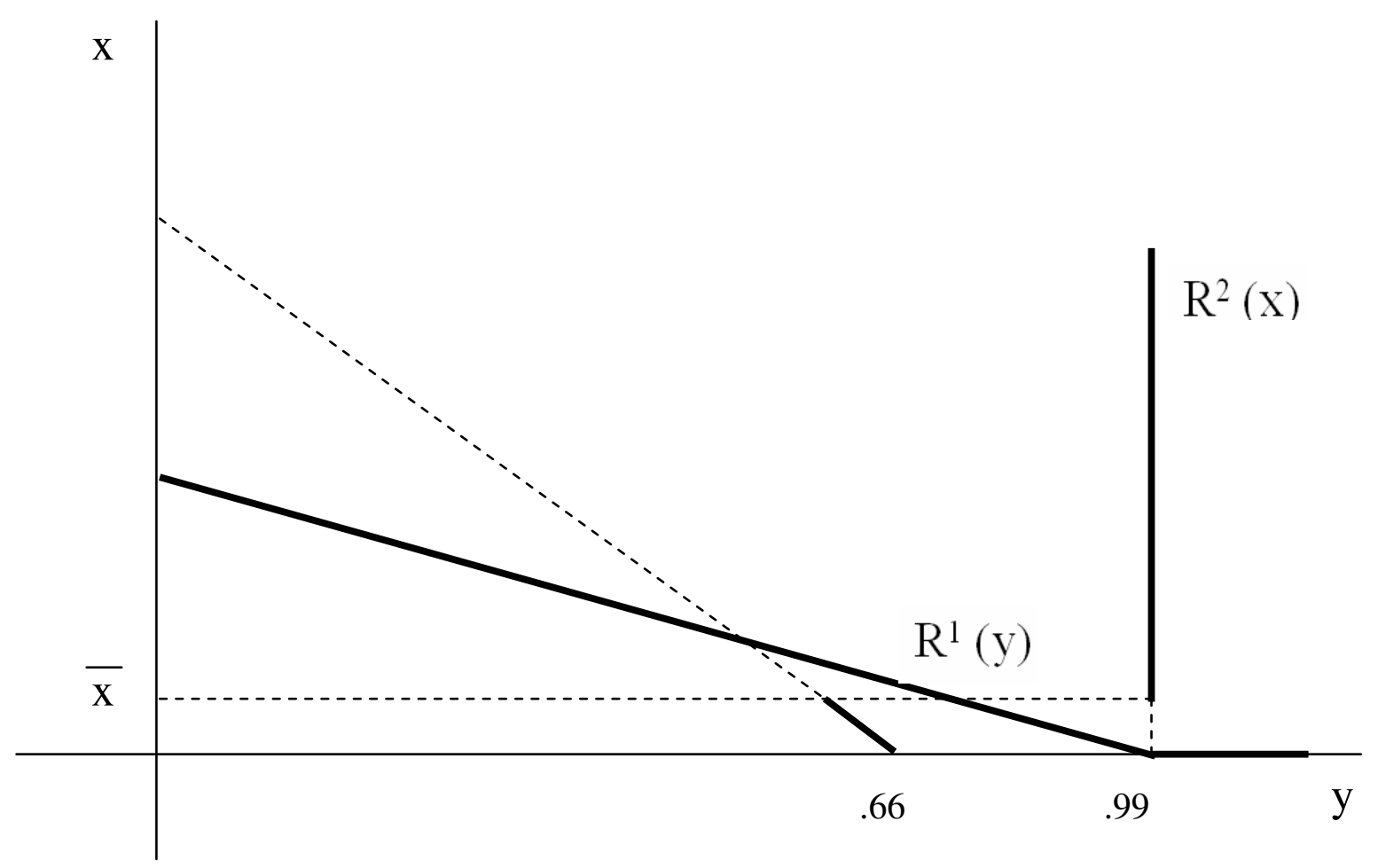

Figure $3 c$ 